LA-13981

Approved for public release;

distribution is unlimited.

Generalizing the Thermodynamics

State Relationships in KIVA-3V 
Los Alamos National Laboratory, an affirmative action/equal opportunity employer, is operated by the University of California for the United States Department of Energy under contract W-7405-ENG-36.

This report was prepared as an account of work sponsored by an agency of the United States Government. Neither the Regents of the University of California, the United States Government nor any agency thereof, nor any of their employees make any warranty, express or implied, or assume any legal liability or responsibility for the accuracy, completeness, or usefulness of any information, apparatus, product, or process disclosed, or represent that its use would not infringe privately owned rights. Reference herein to any specific commercial product, process, or service by trade name, trademark, manufacturer, or otherwise does not necessarily constitute or imply its endorsement, recommendation, or favoring by the Regents of the University of California, the United States Government, or any agency thereof. The views and opinions of authors expressed herein do not necessarily state or reflect those of the Regents of the University of California, the United States Government, or any agency thereof. Los Alamos National Laboratory strongly supports academic freedom and a researcher's right to publish; as an institution, however, the Laboratory does not endorse the viewpoint of a publication or guarantee its technical correctness. 
LA-13981

Issued: October 2002

Generalizing the Thermodynamics

State Relationships in KIVA-3V

Mario F. Trujillo

Peter O'Rourke

David Torres

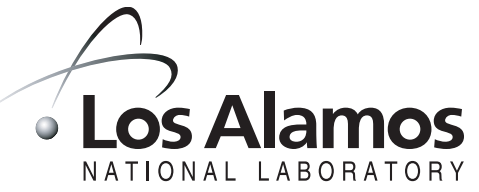





\section{Table Of Contents}

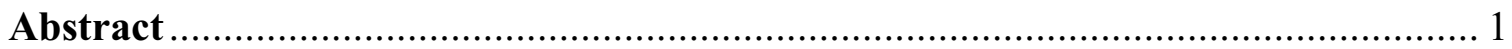

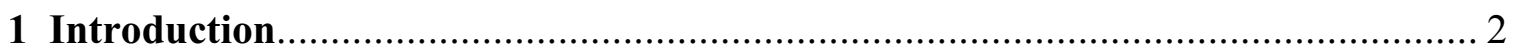

2 The Peng-Robinson Equation of State .......................................................... 2

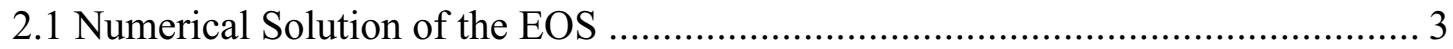

3 Internal Energy Departure from Ideal Behavior ..................................... 4

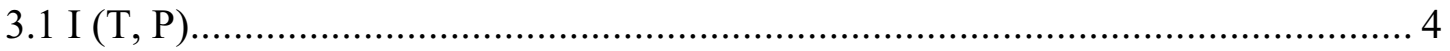

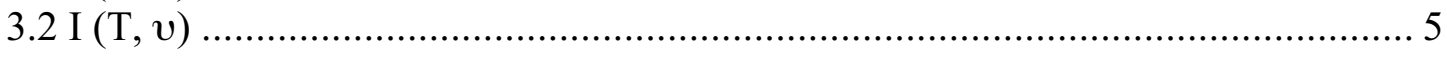

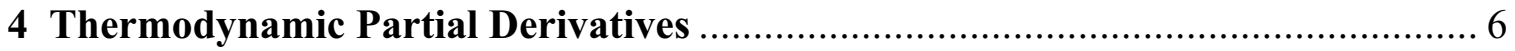

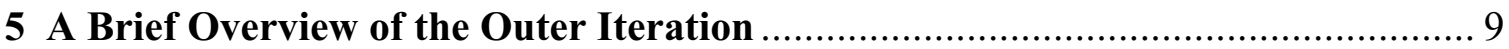

5.1 General Internal Energy Equation............................................................. 10

5.1.1 Special Case of an Ideal Gas .......................................................... 12

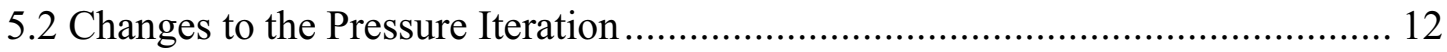

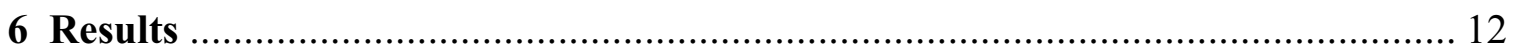

6.1 Cylinder in Compression ........................................................................... 13

6.2 Internal Energy, Specific Heats, and Isentropic Sound Speed.......................... 13

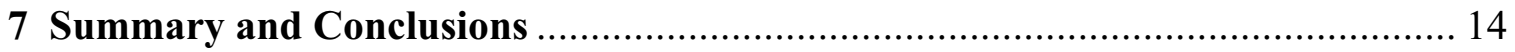

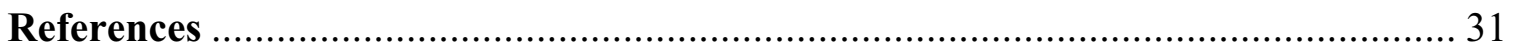

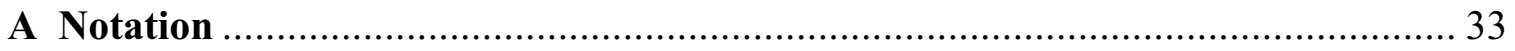

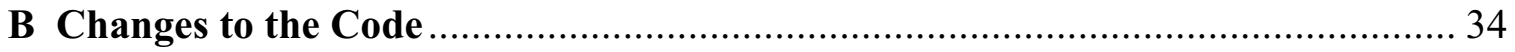

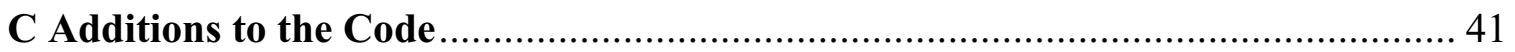




\title{
Generalizing the Thermodynamics State Relationships in KIVA-3V
}

\author{
Mario F. Trujillo, Peter O'Rourke, and David Torres
}

\begin{abstract}
The Peng-Robinson equation of state has been implemented into the KIVA-3V code to better handle high-pressure conditions typical of Diesel engine environments. The implementations modify pressure-volume-temperature relationships, specific heats, and departures in internal energy, among other thermodynamic partial derivatives. Computations show that significant deviations do occur for progressively heavier hydrocarbons. However, when these hydrocarbons exist in a mixture with a non-negligible portion of air, the departures from ideal behavior are mitigated. Internal energy calculations have been extended to allow for pressure effects, but the strongest factor continues to be temperature. Hence departures from ideal behavior in internal energy and related specific heats are minimum.
\end{abstract}




\section{Introduction}

We are interested in employing the KIVA-3V code to simulate physical processes occurring in Diesel environments. One of the major concerns is the assumption of ideal-gas behavior under the high-pressure and -temperature conditions of a Diesel Engine. In addition to affecting P-V-T (Pressure-Volume-Temperature) relationships, the ideal-gas assumption also ignores departures in internal energy at high pressures, changes in important thermodynamic partial derivatives such as specific heats at constant pressure or volume, isentropic sounds speeds, and other thermodynamic derivatives that are used in KIVA-3V. Overall, the ideal-gas assumption currently implemented in KIVA-3V occurs in over 20 subroutines and is basically "hard-wired" into the code.

Our first objective is to remove the limitation of the ideal-gas assumption by introducing an equation of state (EOS) that can more accurately predict thermodynamic quantities at a much wider range of pressures and temperatures. Secondly, we would like to express quantities such as thermodynamic partial derivatives in an abstract form, independent of the type of EOS employed, and to isolate these changes to their own subroutines. This applies also to the calculation of P-V-T relationships. We feel that this approach would facilitate future implementation of other EOS and improve the modularity of the code.

\section{The Peng-Robinson Equation of State}

We have a number of options with regard to choosing a P-V-T relationship, but we desire an EOS form that is both relatively simple and efficient to calculate and also sufficiently accurate. Therefore, we focus on cubic equations of state, given generally as ( [1], p. 43)

$$
P=\frac{R_{u} T}{\bar{v}-b}-\frac{a}{\bar{v}^{2}+\kappa_{1} b \bar{v}+\kappa_{2} b^{2}},
$$

among which we find the van-der-Waals (VDW), Redlich-Kwong (RK), Redlich-Kwong-Soave (RKS), and Peng Robinson (PR). The first and most famous of these four EOS is the VDW (1873). Following the VDW form, the RK EOS was proposed in 1949 significantly improving the accuracy ( [2], p. 393). Nevertheless both $a$ and $b$ were fixed parameters obtained from the critical properties of the substance.

In order to incorporate a temperature dependence on the attractive parameter, $a$, Soave introduced a correction $\alpha(T, \omega)$ that was applied to $a$ as $a=a_{c} \alpha(T, \omega)$ ( [3], p. 79). This modification became known as the RKS EOS. The Peng-Robinson EOS [4] has a similar form, and only deviates from RKS in the value of the constants in the correlations. But by comparison to the RKS, the PR EOS is better at predicting vapor pressures [4] and phase equilibria [5] over a wide range of conditions. Therefore in our work we adopt the PR EOS, which has the form,

$$
P=\frac{R_{u} T}{\bar{v}-b}-\frac{a}{\bar{v}^{2}+2 b \bar{v}-b^{2}}=\frac{R_{u} T}{\bar{v}-b}-\frac{a}{[\bar{v}-b(\sqrt{2}-1)][\bar{v}+b(\sqrt{2}+1)]},
$$

where the parameters $a$ and $b$ (units $F L * L^{3} / M o^{2}$ and $\left(L^{3} / M o\right)$ respectively, Mo is moles) are defined below. The above expression applies only to a pure substance. To extend it for a mixture, the values of $a$ are calculated based on mixing rules ( [1] p. 82), where

$$
a_{m}=\sum_{i} \sum_{j} x_{i} x_{j}\left(a_{i} a_{j}\right)^{1 / 2}\left(1-k_{i j}\right)
$$




$$
b_{m}=\sum_{i} x_{i} b_{i}
$$

The mixture parameters are denoted by subscript $\mathrm{m}$, the mole fractions by $x_{i}$ or $x_{j}$, the pure component parameters by $a_{i}$ or $b_{j}$, and the binary-interaction coefficient by $k_{i j}$. The accentric factor $\left(\omega_{i}\right)$ data comes from tables in Reference 1 and the binary-interaction parameters can be found in Reference 6 . The relations for $a_{i}$ or $b_{j}$ are from Peng-Robinson [4]:

$$
\begin{aligned}
a_{i} & =0.45724 \frac{R_{u}^{2} T_{c, i}^{2}}{P_{c, i}} \times\left[1+f\left(\omega_{i}\right) *\left(1-T_{r, i}^{1 / 2}\right)\right]^{2} ; \\
b_{i} & =0.07780 \frac{R_{u} T_{c, i}}{P_{c}}
\end{aligned}
$$

where $f\left(\omega_{i}\right)=0.37464+1.54226 \omega_{i}-0.26992 \omega_{i}^{2}$, subscript 'c' denotes critical quantities, and $T_{r}$ is the reduced temperature $T / T_{c}$.

\subsection{Numerical Solution of the EOS}

We use a Newton-Raphson method in the solution of the PR EOS. The residual is

$$
R=P-\frac{R_{u} T}{\bar{v}_{m}-b_{m}}+\frac{a_{m}}{\bar{v}_{m}^{2}+2 b \bar{v}_{m}-b_{m}^{2}},
$$

from which we can calculate the derivatives

$$
\frac{d R}{d T}=-\frac{R_{u}}{\bar{v}_{m}-b_{m}}+\frac{d a_{m}}{d T} \frac{1}{\bar{v}_{m}^{2}+2 b \bar{v}_{m}-b_{m}^{2}},
$$

and

$$
\frac{d R}{d \bar{v}_{m}}=\frac{R_{u} T}{\left(\bar{v}_{m}-b_{m}\right)^{2}}-\frac{2 a_{m}\left(\bar{v}_{m}+b_{m}\right)}{\left[\bar{v}_{m}^{2}+2 \bar{v}_{m} b_{m}-b_{m}^{2}\right]^{2}} .
$$

The numerical solution updates guess values of temperature or specific volume, depending on what is being solved, according to

for temperature, and

$$
R\left(T^{n+1}\right)=0=R\left(T^{n}\right)+\frac{d R\left(T^{n}\right)}{d T}\left(T^{n+1}-T^{n}\right)
$$

$$
R\left(\bar{v}_{m}^{n+1}\right)=0=R\left(\bar{v}_{m}^{n}\right)+\frac{d R\left(\bar{v}_{m}^{n}\right)}{d \bar{v}_{m}}\left(\bar{v}_{m}^{n+1}-\bar{v}_{m}^{n}\right)
$$

for specific volume.

Noting the symmetry of $d a_{m} / d T$ above (2), we calculate it as

$$
\frac{d a_{m}}{d T}=\sum_{i} \sum_{j} x_{i} x_{j} a_{i}^{-1 / 2} \frac{d a_{i}}{d T} a_{j}^{1 / 2}\left(1-k_{i j}\right) .
$$

The pure component values of $d a_{i} / d T$ are given by

$$
\begin{aligned}
\frac{d a_{i}}{d T} & =\frac{0.45724 R_{u}^{2} T_{c, i}^{2}}{P_{c, i}} \frac{d}{d T}\left[1+f\left(\omega_{i}\right)\left(1-T_{r}^{1 / 2}\right)\right]^{2} \\
& =\frac{2 \times 0.45724 R_{u}^{2} T_{c, i}^{2}}{P_{c, i}}\left[1+f\left(\omega_{i}\right)\left(1-T_{r}^{1 / 2}\right)\right]\left[-f\left(\omega_{i}\right) \frac{1}{2 \sqrt{T T_{c, i}}}\right] .
\end{aligned}
$$


We observe that for subcritical values of temperature, we may have more than one real root in the PR polynomial. This means that more than one phase is present. We are interested only in the vapor phase, i.e., our efforts are not aimed at simulating condensation/vaporization, which are modeled separately in the treatment of sprays. Also if the pressure is high enough, at subcritical temperature, the only root in the PR polynomial corresponds to the liquid phase. If this occurs, the code does have a problem converging to the solution due to the shape of the residual curve; but even if this is overcome, there is an obvious problem with the presence of only liquid, therefore the code is designed to exit with a warning.

\section{Internal Energy Departure from Ideal Behavior}

In the ideal description of a gas, the internal energy is only a function of temperature. As we move to higher pressures and temperatures, this ideal behavior no longer holds. Therefore, our energy description must incorporate one more independent thermodynamic variable, either $P$ or $v$. We examine both of these representations below.

\section{$3.1 \quad I(T, P)$}

With $I=I(T, P)$ we have

$$
d I=\left.\frac{\partial I}{\partial T}\right|_{P} d T+\left.\frac{\partial I}{\partial P}\right|_{T} d P .
$$

(Note that for an ideal gas $\left.\frac{\partial I}{\partial T}\right|_{P}=\left.\frac{\partial I}{\partial T}\right|_{v}=C_{v}$.) To transform this expression to something we can calculate, let $T d S=d I+P d v$ from the First Law. Taking the derivative of this expression and employing the Maxwell relationship, $\left.\frac{\partial S}{\partial P}\right|_{T}=-\left.\frac{\partial v}{\partial T}\right|_{P}$, yields

$$
-\left.T \frac{\partial v}{\partial T}\right|_{P}=\left.\frac{\partial I}{\partial P}\right|_{T}+\left.\left.P \frac{\partial v}{\partial P}\right|_{T} \rightarrow \frac{\partial I}{\partial P}\right|_{T}=-\left.T \frac{\partial v}{\partial T}\right|_{P}-\left.P \frac{\partial v}{\partial P}\right|_{T} .
$$

We can use the cyclic relation $\left.\left.\left.\frac{\partial v}{\partial T}\right|_{P} \frac{\partial P}{\partial v}\right|_{T} \frac{\partial T}{\partial P}\right|_{v}=-1$, above, to give

$$
\left.\frac{\partial I}{\partial P}\right|_{T}=T \frac{\left.\frac{\partial P}{\partial T}\right|_{v}}{\left.\frac{\partial P}{\partial v}\right|_{T}}-\frac{P}{\left.\frac{\partial P}{\partial v}\right|_{T}} .
$$

This expression is substituted in (7) to give a relation for calculating internal energy differences, i.e.,

$$
d I=\left.\frac{\partial I}{\partial T}\right|_{P} d T+\frac{1}{\left.\frac{\partial P}{\partial v}\right|_{T}}\left[\left.T \frac{\partial P}{\partial T}\right|_{v}-P\right] d P .
$$

Suppose that we are interested in finding the internal energy of a mixture at some $(T, P)$ far removed from ideal-gas behavior. This is done by integrating (8) above:

$$
\begin{aligned}
& I(T, P)-I\left(T_{o}, P_{o}\right)=\left.\int_{T_{o}}^{T} \frac{\partial I}{\partial T}\right|_{P_{o}} d T+\int_{P_{o}}^{P} \frac{1}{\left.\frac{\partial P}{\partial v}\right|_{T}}\left[\left.T \frac{\partial P}{\partial T}\right|_{v}-P\right] d P \\
& I(T, P)-I\left(T_{o}, P_{o}\right)=I\left(T, P_{o}\right)-I\left(T_{o}, P_{o}\right)+\int_{P_{o}}^{P} \frac{1}{\left.\frac{\partial P}{\partial v}\right|_{T}}\left[\left.T \frac{\partial P}{\partial T}\right|_{v}-P\right] d P .
\end{aligned}
$$


Here the reference values are $T_{o}=0 \mathrm{~K}$ and $P_{o}=0$ or $P_{o}=1 \mathrm{~atm}$. The choice of reference pressure is not important since the difference in internal energy at either 1 atm or zero pressure is extremely small. Substituting the PR-EOS to calculate all partial derivatives gives

$$
\begin{aligned}
I(T, P) & =I\left(T, P_{o}\right)+\int_{P_{o}}^{P} \frac{1}{\left.\frac{\partial P}{\partial v}\right|_{T}}\left(\frac{1}{\bar{v}^{2}+2 b_{m} \bar{v}-b_{m}^{2}}\right)\left(a_{m}-T \frac{d a_{m}}{d T}\right) \\
& =I\left(T, P_{o}\right)+\int_{P_{o}}^{P} \frac{1}{\omega_{a v g}}\left(-\frac{R_{u} T}{\left(\bar{v}-b_{m}\right)^{2}}+\frac{2 a_{m}\left(\bar{v}+b_{m}\right)}{\left(\bar{v}^{2}+2 b_{m} \bar{v}-b_{m}^{2}\right)^{2}}\right)^{-1} \\
& \left(\frac{1}{\bar{v}^{2}+2 b_{m} \bar{v}-b_{m}^{2}}\right)\left(a_{m}-T \frac{d a_{m}}{d T}\right) d P
\end{aligned}
$$

which requires a numerical solution. An easier method of computing the internal energy deviation is given next.

\section{$3.2 \quad I(T, v)$}

The other form of describing internal energy, $I=I(T, v)$, gives a differential

$$
d I=\left.\frac{\partial I}{\partial T}\right|_{v} d T+\left.\frac{\partial I}{\partial v}\right|_{T} d v
$$

Employing the First Law, $T d S=d I+P d v$, and the Maxwell relation, $\left.\frac{\partial S}{\partial v}\right|_{T}=\left.\frac{\partial P}{\partial T}\right|_{v}$, gives $\left.T \frac{\partial P}{\partial T}\right|_{v}=$ $\left.\frac{\partial I}{\partial v}\right|_{T}+P$. This is substituted above to yield

$$
d I=\left.\frac{\partial I}{\partial T}\right|_{v} d T+\left(\left.T \frac{\partial P}{\partial T}\right|_{v}-P\right) d v
$$

We would like to integrate this from the reference $\left(T_{o}, v_{o}\right)$ to some point $(T, v)$.

$$
I(T, v)-I\left(T_{o}, v_{o}\right)=I\left(T, v_{o}\right)-I\left(T_{o}, v_{o}\right)+\int_{v_{o}}^{v}\left(\left.T \frac{\partial P}{\partial T}\right|_{v}-P\right) d v
$$

The reference ideal state has an infinite specific volume, i.e., $v_{o}=\infty$. The argument here is probably based on a notion that with infinite specific volume, the molecules are sufficiently far apart that no interaction occurs between them. (Note that we could alternatively define $v_{o}=v\left(P_{o}, T\right)$. The difference between these two quantities is negligible since ideal gas behavior predominates making $\left(\left.T \frac{\partial P}{\partial T}\right|_{v}-P\right) \approx 0$. Returning to the previous equation, we have

$$
I(T, v)=I(T, \infty)+\int_{\infty}^{v}\left(\left.T \frac{\partial P}{\partial T}\right|_{v}-P\right) d v
$$

The first term on the right is obtained from ek () arrays in KIVA-3V, and the second term is calculated using the PR-EOS. The result is

$$
\begin{aligned}
I(T, v) & =I(T, \infty)+\frac{1}{\omega_{a v g}} \int_{\infty}^{\bar{v}} \frac{1}{\bar{v}^{2}+2 b_{m} \bar{v}-b_{m}^{2}}\left(a_{m}-T \frac{d a_{m}}{d T}\right) d \bar{v} \\
& =I(T, \infty)+\left(a_{m}-T \frac{d a_{m}}{d T}\right) \int_{\infty}^{\bar{v}} \frac{d \bar{v}}{\bar{v}^{2}+2 b_{m} \bar{v}-b_{m}^{2}}
\end{aligned}
$$


In closed form the integral can be expressed as

$$
\int \frac{d v}{R}=\frac{1}{\sqrt{-\Delta}} \ln \left|\frac{\sqrt{-\Delta}-(b+2 c v)}{(b+2 c v)+\sqrt{-\Delta}}\right|,
$$

where

$$
\begin{aligned}
R & =a+b v+c v^{2}, \quad a=-b_{m}^{2}, \quad b=2 b_{m}, \\
c & =1, \text { and } \quad \Delta=4 a c-b^{2}=-8 b_{m}^{2} .
\end{aligned}
$$

Substituting this into (11) gives

$$
I(T, v)=I(T, \infty)+\left(\frac{a_{m}-T \frac{d a_{m}}{d T}}{\omega_{\text {avg }} 2 \sqrt{2} b_{m}}\right) \times\left[\ln \left|\frac{b_{m}(\sqrt{2}-1)-\bar{v}}{b_{m}(\sqrt{2}+1)+\bar{v}}\right|\right] .
$$

\section{Thermodynamic Partial Derivatives}

Employing the PR-EOS and the thermodynamic tables available in KIVA-3V, we can readily compute specific heats, isentropic sound speeds, and other thermodynamic partial derivatives, which are used throughout the code. In this section, we summarize their mathematical form. The PR coefficients $a_{m}, b_{m}$, and the derivatives $d a_{m} / d T$ are computed from Eqs. (2), (3), and (6) respectively.

- $\left.\frac{\partial P}{\partial T}\right|_{\rho}$

Using the PR-EOS, we get

$$
\left.\frac{\partial P}{\partial T}\right|_{\rho}=\left.\frac{\partial P}{\partial T}\right|_{\bar{v}}=\frac{R_{u}}{\bar{v}-b}-\frac{d a_{m}}{d T} \frac{1}{\bar{v}^{2}+2 b_{m} \bar{v}-b_{m}^{2}} .
$$

- $C_{T}^{2}=\left.\frac{\partial P}{\partial \rho}\right|_{T}$

Substituting the PR-EOS gives

$$
\begin{aligned}
\left.\frac{\partial P}{\partial \rho}\right|_{T} & =\left.\frac{\partial P}{\partial \bar{v}} \frac{\partial \bar{v}}{\partial v} \frac{\partial v}{\partial \rho}\right|_{T} ; \\
C_{T}^{2} & =\left[\frac{-R_{u} T}{\left(\bar{v}-b_{m}\right)^{2}}+\frac{2 a_{m}\left(\bar{v}+b_{m}\right)}{\left(\bar{v}^{2}+2 b_{m} \bar{v}-b_{m}^{2}\right)^{2}}\right] \frac{-\omega_{\text {avg }}}{\rho^{2}} .
\end{aligned}
$$

- $\left.\frac{\partial I}{\partial T}\right|_{P}$

The internal energy corresponding to a fixed composition is a function of two independent variables, e.g., $I(T, \rho)=I(T, P)$. Hence the differential

$$
d I=\left.\frac{\partial I}{\partial T}\right|_{\rho} d T+\left.\frac{\partial I}{\partial \rho}\right|_{T} d \rho=\left.\frac{\partial I}{\partial T}\right|_{P} d T+\left.\frac{\partial I}{\partial P}\right|_{T} d P
$$

and if we are considering a change in internal energy occurring at constant pressure then

$$
\left.\frac{\partial I}{\partial T}\right|_{P} d T=\left.\frac{\partial I}{\partial T}\right|_{\rho} d T+\left.\frac{\partial I}{\partial \rho}\right|_{T} d \rho
$$


We can similarly write the differential for $\rho(T, P)$ as

$$
d \rho=\left.\frac{\partial \rho}{\partial T}\right|_{P} d T+\left.\frac{\partial \rho}{\partial P}\right|_{T} d P
$$

and introduce it above, at constant $P$, to yield

$$
\left.\frac{\partial I}{\partial T}\right|_{P}=\left.\frac{\partial I}{\partial T}\right|_{\rho}+\left.\left.\frac{\partial I}{\partial \rho}\right|_{T} \frac{\partial \rho}{\partial T}\right|_{P} .
$$

From the cyclical relationship

$$
\left.\left.\left.\frac{\partial \rho}{\partial T}\right|_{P} \frac{\partial P}{\partial \rho}\right|_{T} \frac{\partial T}{\partial P}\right|_{\rho}=\left.\left.\frac{\partial \rho}{\partial T}\right|_{P} C_{T}^{2} \frac{\partial T}{\partial P}\right|_{\rho}=-1,
$$

we can also express this as

$$
\left.\frac{\partial I}{\partial T}\right|_{P}=C_{v}-\left.\left.\frac{\partial I}{\partial \rho}\right|_{T} \frac{\partial P}{\partial T}\right|_{\rho} \frac{1}{C_{T}^{2}} .
$$

In the code, we compute this as

$$
\left.\frac{\partial I}{\partial T}\right|_{P}=\frac{I[T+\Delta T, v(T+\Delta T, P)]-I[T, v(T, P)]}{\Delta T},
$$

where $\Delta T$ is $100 \mathrm{~K}$, and the internal energies are calculated using Eq. (12).

- Isentropic sound speed, $C_{s}^{2}=\left.\frac{\partial P}{\partial \rho}\right|_{S}$

We regard $P(\rho, T)=P(\rho, S)$ and write the differential

$$
d P=\left.\frac{\partial P}{\partial \rho}\right|_{T} d \rho+\left.\frac{\partial P}{\partial T}\right|_{\rho} d T=\left.\frac{\partial P}{\partial S}\right|_{\rho} d S+\left.\frac{\partial P}{\partial \rho}\right|_{S} d \rho .
$$

For an isentropic process, this reduces to

$$
\left.\frac{\partial P}{\partial \rho}\right|_{T} d \rho+\left.\frac{\partial P}{\partial T}\right|_{\rho} d T=\left.\frac{\partial P}{\partial \rho}\right|_{S} d \rho .
$$

Combining this with the differential of $T(\rho, S)$,

$$
d T=\left.\frac{\partial T}{\partial \rho}\right|_{S} d \rho+\left.\frac{\partial T}{\partial S}\right|_{\rho} d S,
$$

evaluated for $d S=0$, and substituting it above gives

$$
\left.\frac{\partial P}{\partial \rho}\right|_{T}+\left.\left.\frac{\partial P}{\partial T}\right|_{\rho} \frac{\partial T}{\partial \rho}\right|_{S}=\left.\frac{\partial P}{\partial \rho}\right|_{S} .
$$

Employing again a cyclical relation for $T, S$, $\rho$, i.e.,

$$
\left.\left.\left.\frac{\partial T}{\partial \rho}\right|_{S} \frac{\partial S}{\partial T}\right|_{\rho} \frac{\partial \rho}{\partial S}\right|_{T}=-1
$$


yields

$$
\left.\frac{\partial P}{\partial \rho}\right|_{S}=\left.\frac{\partial P}{\partial \rho}\right|_{T}-\left.\frac{\partial P}{\partial T}\right|_{\rho} \frac{\partial S /\left.\partial \rho\right|_{T}}{\partial S /\left.\partial T\right|_{\rho}}
$$

We would like to clean up the far right term. From the First Law, $\left.T \frac{\partial S}{\partial T}\right|_{\rho}=C_{v}$. We can equate $S(v, T)=$ $S(\rho, T)$ and compute the differential to yield $\left.\frac{\partial S}{\partial v}\right|_{T} d v=\left.\frac{\partial S}{\partial \rho}\right|_{T} d \rho$ or

$$
-\left.\frac{1}{\rho^{2}} \frac{\partial S}{\partial v}\right|_{T}=\left.\frac{\partial S}{\partial \rho}\right|_{T}
$$

One of Maxwell's relations is

$$
\left.\frac{\partial P}{\partial T}\right|_{\rho}=\left.\frac{\partial S}{\partial v}\right|_{T}
$$

which gives

$$
\left.\frac{\partial S}{\partial \rho}\right|_{T}=-\left.\frac{1}{\rho^{2}} \frac{\partial P}{\partial T}\right|_{\rho}
$$

Substituting back into (\#) results

$$
C_{s}^{2}=\left.\frac{\partial P}{\partial \rho}\right|_{S}=\left.\frac{\partial P}{\partial \rho}\right|_{T}+\frac{T}{C_{v} \rho^{2}}\left(\left.\frac{\partial P}{\partial T}\right|_{\rho}\right)^{2} .
$$

\section{- Constant pressure specific heat, $C_{p}$}

By definition, $C_{p}=\left.\frac{\partial H}{\partial T}\right|_{P}$. From the First Law and the definition of enthalpy, we get $T d S=d H-v d P$, which gives $C_{p}=\left.T \frac{\partial S}{\partial T}\right|_{P}$. Entropy can be written as $S(T, P)=S(T, \rho)$; consequently at constant pressure, the differential of these quantities results in

$$
\begin{aligned}
\left.\frac{\partial S}{\partial T}\right|_{P} d T & =\left.\frac{\partial S}{\partial T}\right|_{\rho} d T+\left.\frac{\partial S}{\partial \rho}\right|_{T} d \rho \\
& =\left.\frac{\partial S}{\partial T}\right|_{\rho} d T+\left.\frac{\partial S}{\partial \rho}\right|_{T}\left\{\left.\frac{\partial \rho}{\partial T}\right|_{P} d T+\left.\frac{\partial \rho}{\partial P}\right|_{T} d P\right\} ; \\
\left.T \frac{\partial S}{\partial T}\right|_{P} & =\left.T \frac{\partial S}{\partial T}\right|_{\rho}+\left.\left.T \frac{\partial S}{\partial \rho}\right|_{T} \frac{\partial \rho}{\partial T}\right|_{P} ; \\
C_{p} & =C_{v}+\left.\left.T \frac{\partial S}{\partial \rho}\right|_{T} \frac{\partial \rho}{\partial T}\right|_{P} \\
C_{p} & =C_{v}-\left.\left.\frac{T}{\rho^{2}} \frac{\partial P}{\partial T}\right|_{\rho} \frac{\partial \rho}{\partial T}\right|_{P}
\end{aligned}
$$

The last step here comes from (\&). Substituting the cyclic relationship

$$
-\left.\frac{\partial P}{\partial T}\right|_{\rho}\left(\left.\frac{\partial P}{\partial \rho}\right|_{T}\right)^{-1}=\left.\frac{\partial \rho}{\partial T}\right|_{P}
$$

above, gives

$$
C_{p}=C_{v}+\frac{T}{\rho^{2}}\left(\left.\frac{\partial P}{\partial T}\right|_{\rho}\right)^{2}\left(\left.\frac{\partial P}{\partial \rho}\right|_{T}\right)^{-1} .
$$


- $\left.\frac{\partial V}{\partial T}\right|_{P}$

The specific volume, $v=V / m$, can be introduced into the cyclic relationship

$$
\left.\left.\left.\frac{\partial v}{\partial T}\right|_{P} \frac{\partial P}{\partial v}\right|_{T} \frac{\partial T}{\partial P}\right|_{v}=-1
$$

to give

$$
\left.\frac{\partial V}{\partial T}\right|_{P}=\frac{V}{\rho} \frac{\left.\frac{\partial P}{\partial T}\right|_{\rho}}{\left.\frac{\partial P}{\partial \rho}\right|_{T}} .
$$

- Constant Volume Specific Heat, $C_{v}$

From definition $C_{v}=\left.\frac{\partial I}{\partial T}\right|_{v}$, the specific heat is calculated using the expression

$$
C_{v}=\frac{I(T+\Delta T, v)-I(T, v)}{\Delta T},
$$

where $I(T+\Delta T, v)$ and $I(T, v)$ are computed from Eq. (12) and $\Delta T=100 K$, since the table entries in fuelib.f are in 100-degree intervals.

- Verifying $C_{s}^{2}=\gamma C_{T}^{2}$

We would like to verify $C_{s}^{2}=\gamma C_{T}^{2}$ :

$$
\begin{aligned}
\gamma & =\frac{C_{p}}{C_{v}}=1+\frac{T}{\rho^{2} C_{v}}\left(\left.\frac{\partial P}{\partial T}\right|_{\rho}\right)^{2}\left(\left.\frac{\partial P}{\partial \rho}\right|_{T}\right)^{-1} \\
& =1+\frac{T}{\rho^{2} C_{v}}\left(\left.\frac{\partial P}{\partial T}\right|_{\rho}\right)^{2}\left(C_{T}^{2}\right)^{-1} ; \\
\gamma C_{T}^{2} & =C_{T}^{2}+\frac{T}{\rho^{2} C_{v}}\left(\left.\frac{\partial P}{\partial T}\right|_{\rho}\right)^{2} ; \\
\gamma C_{T}^{2} & =C_{s}^{2} .
\end{aligned}
$$

\section{A Brief Overview of the Outer Iteration}

The central part of the KIVA-3V code is the gas-flow solver. On top of this part, the models for sprays, chemical reactions, impingement processes, etc., are built. The variables that are computed in the gas-flow part are the species densities, $\rho_{m}$, the turbulent kinetic energy and dissipation, $k$ and $\varepsilon$, the velocity field $\mathbf{u}$, the temperature $T$, the cell volumes $V$, the pressure $P$, and related quantities. Since the equations governing the relationship of these variables are typically interwoven with one another, iteration procedures are used to arrive at solutions to these equations.

The outer iteration consists of the computation of the velocity, temperature, cell volume, and pressure. Subset to this are smaller iterations for specific variables. Turbulent kinetic energy, dissipation, and species densities do not enter into the outer iteration because they are weakly linked to the former terms.

The steps outlining the outer iteration are as follows: 
1. Initial guess on the pressure using Eq. (108) of the KIVA-II manual.

2. Calculation of the velocity field based on Eq.(78) of the KIVA-II manual. Notice that the pressure, $P^{B}$, on the r.h.s corresponds to the pressure calculated in step 1 .

3. The temperature is solved using Eq. (110) of the KIVA-II manual or in the general case of a real gas Eq. (28) derived in this paper. In this step, the pressure and the velocities computed previously for the ${ }^{B}$ level are used.

4. Based on this temperature and pressure and knowledge of the mass, which come from solving the species density equation, the cell volume, $V^{p}$, is computed, employing Eq. (111).

5. This cell volume is typically different than the cell volume calculated in Eq. (102), i.e.,

$$
V^{B}=V^{c}=V^{n}+\Delta t \sum_{a}(u A)_{a}^{B} .
$$

To bring both of these volumes to a sufficiently similar value, a relationship is needed between volume and pressure. The objective is to modify the pressure with the intent of reducing the gap between the volumes. The relationship is

$$
V^{c}=V^{p}+\left.\frac{\partial V^{p}}{\partial P}\right|_{S}\left(P^{c}-P^{p}\right)
$$

from which the unknown $P^{c}$ is solved for. The value of $P^{c}-P^{p}$ is checked to determine if convergence as been achieved. Eq. (86) is used to modify the fluxes $u A$ in this iteration.

6. If $P^{c}-P^{p}$ is too large, then $P^{p}=P^{c}$ and we return to step 2 .

\subsection{General Internal Energy Equation}

The subscripts $i j k$ on field variables are omitted.

The central energy equation solved in KIVA-3V is Eq. (89) of the KIVA-II manual, shown below. It represents a process of heat and mass addition (the latter occurs in the presence of vaporizing spray) that takes place under variable pressure and volume.

$$
\begin{aligned}
\frac{M^{B} I^{B}-M^{n} I^{n}}{\Delta T} & =-\left(\frac{P^{n}+P^{B}}{2}\right)\left(\frac{V^{B}-V^{n}}{\Delta t}\right)+\left(1-A_{o}\right)\left[\phi_{D} \sigma^{B}: \nabla \mathbf{u}^{B}+\left(1-\phi_{D}\right) \sigma^{n}: \nabla \mathbf{u}^{n}\right] V^{n} \\
& +A_{o} M^{B} \epsilon^{A}+\sum_{a}(\rho D)_{a}{ }^{n}\left[\sum_{n} h_{m} T_{a}^{n} \nabla\left(\phi_{D} Y_{m}^{B}+\left(1-\phi_{D}\right) Y_{m}^{A}\right)_{a}\right] \cdot \mathbf{A}_{a}^{n} \\
& +\sum_{a} K_{a}^{n} \nabla\left[\phi_{D} T^{B}+\left(1-\phi_{D}\right) \tilde{T}\right]_{a} \cdot \mathbf{A}_{a}^{n}+V^{n}\left(\dot{Q}^{s}+\dot{Q}^{c}\right) .
\end{aligned}
$$

This equation is solved in a sequence of three steps, which are denoted here as (S1), (S2), and (S3). Each of these steps is done at either constant volume or constant pressure.

The first step is from the n-level discretization to the A-phase, and it proceeds at constant volume:

$$
\frac{M^{A} I^{A}-M^{n} I^{n}}{\Delta t}=V^{n}\left(\dot{Q}^{s}+\dot{Q}^{c}\right)
$$


The second step is from the A-phase to the t-state:

$$
M^{B} \frac{I^{t}-I^{A}}{\Delta t}=A_{o} M^{B} \epsilon^{A}+\sum_{a}(\rho D)_{a}{ }^{n}\left[\sum_{n} h_{m} T_{a}^{n} \nabla\left(\phi_{D} Y_{m}^{B}+\left(1-\phi_{D}\right) Y_{m}^{A}\right)_{a}\right] \cdot \mathbf{A}_{a}^{n}
$$

The last step occurs at constant pressure, and it is from the t-state to the B-phase:

$$
\begin{aligned}
M^{B} \frac{I^{B}-I^{t}}{\Delta t} & =-\left.P \frac{\Delta V}{\Delta t}\right|_{t} ^{B}+\left(1-A_{o}\right)\left[\phi_{D} \sigma^{B}: \nabla \mathbf{u}^{B}+\left(1-\phi_{D}\right) \sigma^{n}: \nabla \mathbf{u}^{n}\right] V^{n} \\
& +\sum_{a} K_{a}^{n} \nabla\left[\phi_{D} T^{B}+\left(1-\phi_{D}\right) \tilde{T}\right]_{a} \cdot \mathbf{A}_{a}^{n}
\end{aligned}
$$

where $\tilde{T}$ is given in Eq. (94) of the Kiva manual and

$$
-\left.P \frac{\Delta V}{\Delta t}\right|_{t} ^{B}=-\left(\frac{P^{n}+P^{B}}{2}\right)\left(\frac{V^{B}-V^{n}}{\Delta t}\right) .
$$

In our current efforts to generalize the caloric equation of state in KIVA-3V, we concentrate on the last step. First, the differentials $d I$ and $d V$, hinted by (S3), can be expressed as

$$
I^{B}-I^{t}=\left.\frac{\partial I}{\partial T}\right|_{P}\left(T^{B}-T^{t}\right)
$$

and

$$
V^{B}-V^{n}=\left(V^{B}-V^{t}\right)+\left(V^{t}-V^{n}\right)=\left.\frac{\partial V}{\partial T}\right|_{P}\left(T^{B}-T^{t}\right)+\left(V^{t}-V^{n}\right),
$$

since Eq. (S3) represents a constant pressure process.

Second, for brevity in notation, we define

$$
\mathfrak{D}_{i f f}=\sum_{a} K_{a}^{n} \nabla\left\{\phi_{D} T^{B}+\left(1-\phi_{D}\right) \tilde{T}\right\}_{a} \cdot \mathbf{A}_{a}^{n}
$$

and

$$
\mathcal{D}_{i s s}=\left[\phi_{D} \sigma^{B}: \nabla \mathbf{u}^{B}+\left(1-\phi_{D}\right) \sigma^{n}: \nabla \mathbf{u}^{n}\right] V^{n} .
$$

Substituting (23), (24), (25), (26), and (27) into (S3), we get

$$
\left.\frac{M^{B}}{\Delta t} \frac{\partial I}{\partial T}\right|_{P}\left(T^{B}-T^{t}\right)=-\left(\frac{P^{n}+P^{B}}{2 \Delta t}\right)\left[\left.\frac{\partial V}{\partial T}\right|_{P}\left(T^{B}-T^{t}\right)+\left(V^{t}-V^{n}\right)\right]+\mathfrak{D}_{i f f}+\left(1-A_{o}\right) \mathcal{D}_{i s s} .
$$

Multiplying through by $\frac{\Delta t}{\left.M^{B} \frac{\partial I}{\partial T}\right|_{P}}$ and manipulating the equation we get

$$
T^{B}=\frac{T^{t}+\left(\frac{P^{n}+P^{B}}{\left.2 M^{B} \frac{\partial I}{\partial T}\right|_{P}}\right)\left[\left.\frac{\partial V}{\partial T}\right|_{P} T^{t}-\left(V^{t}-V^{n}\right)\right]+\frac{\Delta t}{\left.M^{B} \frac{\partial I}{\partial T}\right|_{P}}\left(\mathfrak{D}_{i f f}+\left(1-A_{o}\right) \mathcal{D}_{i s s}\right)}{\left[1+\left.\left(\frac{P^{n}+P^{B}}{\left.2 M^{B} \frac{\partial I}{\partial T}\right|_{P}}\right) \frac{\partial V}{\partial T}\right|_{P}\right]} .
$$




\subsubsection{Special case of an ideal gas}

In the case we have an ideal gas, then $\left.\frac{\partial I}{\partial T}\right|_{P}=C_{v}$ and $\left.\frac{\partial V}{\partial T}\right|_{P}=\frac{M^{B} \bar{R}}{P}$; also $\left.\frac{\partial V}{\partial T}\right|_{P} T^{t}=V^{t}$. Substituting above,

$$
T_{\text {ideal }}^{B}=\frac{T^{t}+\left(\frac{P^{n}+P^{B}}{2 M^{B} C_{v}}\right)\left[V^{n}\right]+\frac{\Delta t}{M^{B} C_{v}}\left(\mathfrak{D}_{i f f}+\left(1-A_{o}\right) \mathcal{D}_{i s s}\right)}{\left[1+\left(\frac{P^{n}+P^{B}}{2 M^{B} C_{v}}\right) \frac{M^{B} \bar{R}}{P}\right]} .
$$

This is the original expression which appears in the KIVA-II manual.

\subsection{Changes to the Pressure Iteration}

The pressure correction currently done in KIVA-3V, and presented in Eq. (112) is

$$
V^{c}=V^{p}-\frac{V^{p}}{\gamma P^{p}}\left(P^{c}-P^{p}\right)
$$

where $V^{c}$ is calculated by Eq. (102),

$$
V^{c}=V^{n}+\Delta t \sum_{a}(u A)_{a}^{B} .
$$

Since the volumes $V^{p}$ and $V^{c}$ are typically different in the first iterations, the equation above is used to modify the pressures, which gives rise to a new value of $V^{p}$. The modified or corrected pressures also change the values of $u A$ through Eq. (82) of the KIVA-II manual, and hence $V^{c}$. The iteration continues until the pressures and volumes are sufficiently close.

In the generalization of the code, the isentropic relationship between volume and pressure (30) is changed to

$$
V^{c}=V^{p}+\left.\frac{\partial V^{p}}{\partial P}\right|_{S}\left(P^{c}-P^{p}\right)
$$

Since $V=M / \rho$, we have that

or

$$
\left.\frac{\partial V^{p}}{\partial P}\right|_{S}=-\left.\frac{M}{\rho^{2}} \frac{\partial \rho^{p}}{\partial P}\right|_{S}=-\left.\frac{V}{\rho} \frac{\partial \rho^{p}}{\partial P}\right|_{S}
$$

$$
\left.\frac{\partial V^{p}}{\partial P}\right|_{S}=-\frac{V}{\rho} \frac{1}{C_{s}^{2}}
$$

\section{Results}

We are interested in examining the departure from ideal behavior in quantities such as PVT relationship, thermodynamic partial derivatives, and caloric quantities. The first cases studied correspond to a cylinder in compression with temperature and pressure conditions typical of Diesel engine environments. The calculations presented next correspond to departures in internal energy, specific heat, and isentropic sound speed and represent a select number of all the thermodynamic partial derivatives that were modified to the PR EOS form. 


\subsection{Cylinder in Compression}

Four different gaseous mixtures undergoing compression in a typical engine geometry are studied. The first mixture is composed of pure air, the second mixture contains $75 \% \mathrm{~N}_{2}$ and $25 \% \mathrm{C}_{10} \mathrm{H}_{8}$, the third mixture is made up of $25 \% \mathrm{~N}_{2}$ and $75 \% \mathrm{C}_{10} \mathrm{H}_{8}$, and the final mixture is $5 \% \mathrm{~N}_{2}$ and $95 \% \mathrm{C}_{10} \mathrm{H}_{8}$. These compositions were chosen to examine the effect of hydrocarbon addition in decreasing or increasing the departure from ideal gas behavior. In order to measure such departure, the compressibility factor defined as

$$
Z=\frac{P \bar{v}}{R_{u} T}
$$

is used, which shows that the closer $Z$ is to 1.0, the closer the gas behavior is to ideal. The initial conditions for all compression cases are as follows: temperature $=620 \mathrm{~K}$, pressure $=0.175 \mathrm{MPa}$, and a compression ratio of 15.1. The results are shown in Figures 1 through 8 in terms of the Z-factor at both the bottom center position, where pressure is not significantly large, and the top center position, where it is expected that the greatest deviation from ideal behavior occurs as a consequence of the maximum pressure.

If we consider the pure-air case first, the value of $Z$ is 1.0 at bottom center (Fig.1) and then increases slightly by the time the piston reaches top center (Fig.2). Since the deviation is approximately $1 \%$, it can be concluded that air behaves like an ideal gas. In the next pair of Figures (Figs. 3 and 4), air has been replaced with pure nitrogen, and $25 \% \mathrm{C}_{10} \mathrm{H}_{8}$ has been introduced. At bottom center, the Z-factor is still at 1.0 and at top center the values are very close to the ones predicted for pure air. If we increase the hydrocarbon content to $75 \% \mathrm{C}_{10} \mathrm{H}_{8}$ and $25 \% \mathrm{~N}_{2}$, then the results show a neglibible departure from 1.0 at bottom center (Fig.5). As the mixture is compressed the deviation from $Z=1$ becomes approximately $4 \%$ (Fig.6). If we continue to increase the hydrocarbon content by nearly displacing all of the $N_{2}$, e.g. $95 \% C_{10} H_{8}$ and $5 \%$ $N_{2}$, the values for $Z$ are around 0.98 at bottom center (Fig.7) and approximately 0.80 at top center (Fig.8). The deviation becomes significant with an increasing presence of the hydrocarbon, particularly in the cooler regions of the domain, e.g., near the walls.

This trend is explained by studying the compressibility chart shown in Figure 9. For pure air, which is composed mainly of nitrogen and then oxygen, the critical temperatures are respectively $126 \mathrm{~K}$ and $154.5 \mathrm{~K}$ and the critical pressures are 3.39 and $5.05 \mathrm{MPa}$. This gives reduced temperatures $\left(T / T_{c}\right)$ that are beyond 4.0 at bottom center and continue to increase as the cylinder undergoes compression. By examining the compressibility chart, this places air in a path of slightly increasing $Z$ with pressure. In contrast to either nitrogen or oxygen, hydrocarbons have typically much higher critical temperatures, e.g., $C_{10} H_{8} T_{c}=748.4$, which results in reduced temperatures in the range between 1.0 and 2.0. Hence, as they are compressed, the path that they follow is one of decreasing $Z$, and the closer that path is to the isotherm $T / T_{c}=1.0$, the more drastic the deviation from ideal behavior. This helps to understand the reason for the greater decrease in $Z$ in the cooler regions near the walls (e.g., see Figure 8). (Note that major deviations in $Z$ can occur for air at pressures 10 times the critical pressure, but these conditions are not reached during typical diesel engines conditions.)

\subsection{Internal Energy, Specific Heats, and Isentropic Sound Speed}

In the following plots (Figures 10- 21), we examine the departure from ideal behavior of some thermodynamic quantities. Our results are presented as a function of temperature for three different pressures, namely $0.1 \mathrm{MPa}, 2.0 \mathrm{MPa}$, and 5.0 MPa. The predictions at $0.1 \mathrm{MPa}(\approx 1 \mathrm{~atm})$ can be interpreted as pertaining to ideal-gas behavior. 
First, we look at deviations of internal energy caused by pressure. Our calculations are presented in terms of the change in internal energy between an ideal state and a compressed state normalized by the internal energy of the ideal state. A given temperature, $\mathrm{T}$, and an infinite specific volume specify the ideal state, and the same temperature but a much lower specific volume defines the compressed state. The infinite value of the specific volume guarantees no molecular interactions. (It should be noted that the difference between this ideal state and the one defined by a state having the same temperature but a specific volume corresponding to standard pressure is insignificant.)

The results presented in Figures 10, 11, and 12 correspond to air, $C_{4} H_{10}$, and $C_{10} H_{22}$, respectively. The trend is the same for all three gases. It consists of a more pronounced deviation at lower temperature that gradually decreases as the temperature is increased; and as expected, the departure is greater with higher pressure. The tapering of the internal energy departure is due to an increase in the normalizing internal energy with temperature and also to a closer proximity to the $Z=1$ line as we move in the compressibity chart in the direction of higher temperature at a fixed pressure. Physically, this corresponds to a greater distance between gas molecules with higher temperatures which reduces the interaction between them and hence approaches ideal behavior. The difference among the three gases is in the magnitude of the deviation, which for air is approximately $1 \%$ maximum, and for the heavier gas $C_{10} H_{22}$, is around $4 \%$.

The next set of calculations is for constant volume specific heat, $C_{v}$, plotted as a function of temperature for increasing values of pressure. The results for air, $C_{4} H_{10}$, and $C_{10} H_{22}$ are shown respectively in Figures 13, 14, and 15. Since the internal energy departures were relatively insignificant as shown in the previous plots and the specific heats are derived from internal energy behavior, it is no surprise to see that all three lines representing the different pressures fall almost on top of one another. Air, due to its low critical temperature, shows the lowest sensitivity to pressure changes.

Related to the specific heat calculation is the calculation for $\gamma$, the specific heat ratio. The results are presented in Figure 16, for air, Figure 17 for $\mathrm{C}_{4} \mathrm{H}_{10}$, and Figure 18 for $\mathrm{C}_{10} \mathrm{H}_{22}$. The deviations are once again more noticeable for the heavier hydrocarbons by contrast to the almost negligible effect for air. For all three mixtures, the influence of pressure is mitigated as one moves to higher temperatures, as previously shown.

The last set of plots illustrates the behavior of the isentropic sound speed, shown in Figures 19, 20, and 21 for air, $C_{4} H_{10}$, and $C_{10} H_{22}$, respectively. The ideal result gives $C_{s}=\sqrt{\gamma R T}$, and for air this behavior is insignificantly altered as one moves to higher pressures. By contrast, $C_{10} H_{22}$ displays large differences, particularly at lower temperatures where it has been explained that large deviations from ideal behavior are likely to occur. The $C_{4} H_{10}$ case falls in between air and the heavier hydrocarbon, as expected.

\section{Summary and Conclusions}

The thermodynamic relationships in KIVA-3V have been modified from the ideal to a Peng-Robinson EOS to better predict quantities at high pressures typical of Diesel environments. The changes involve PVT relationships, internal energy departures from ideal behavior, specific heats, isentropic sound speed, and other thermodynamic partial derivatives that are used, for instance, in the internal energy calculation. A number of computations have been presented which illustrate the effect of high pressure on the departure from ideal behavior. The main conclusion from these results is that hydrocarbons, particularly heavier hydrocarbons with high critical temperatures, exhibit significant deviations from ideal behavior. This is 
reflected mostly in the PVT relationship, where the compressibility factor is used to measure the deviation, but not so significantly in departures of internal energy or in quantities derived from the internal energy (e.g., specific heats). Moreover, the presence of air in mixture with hydrocarbons has a strong tendency to diminish the real gas behavior. 

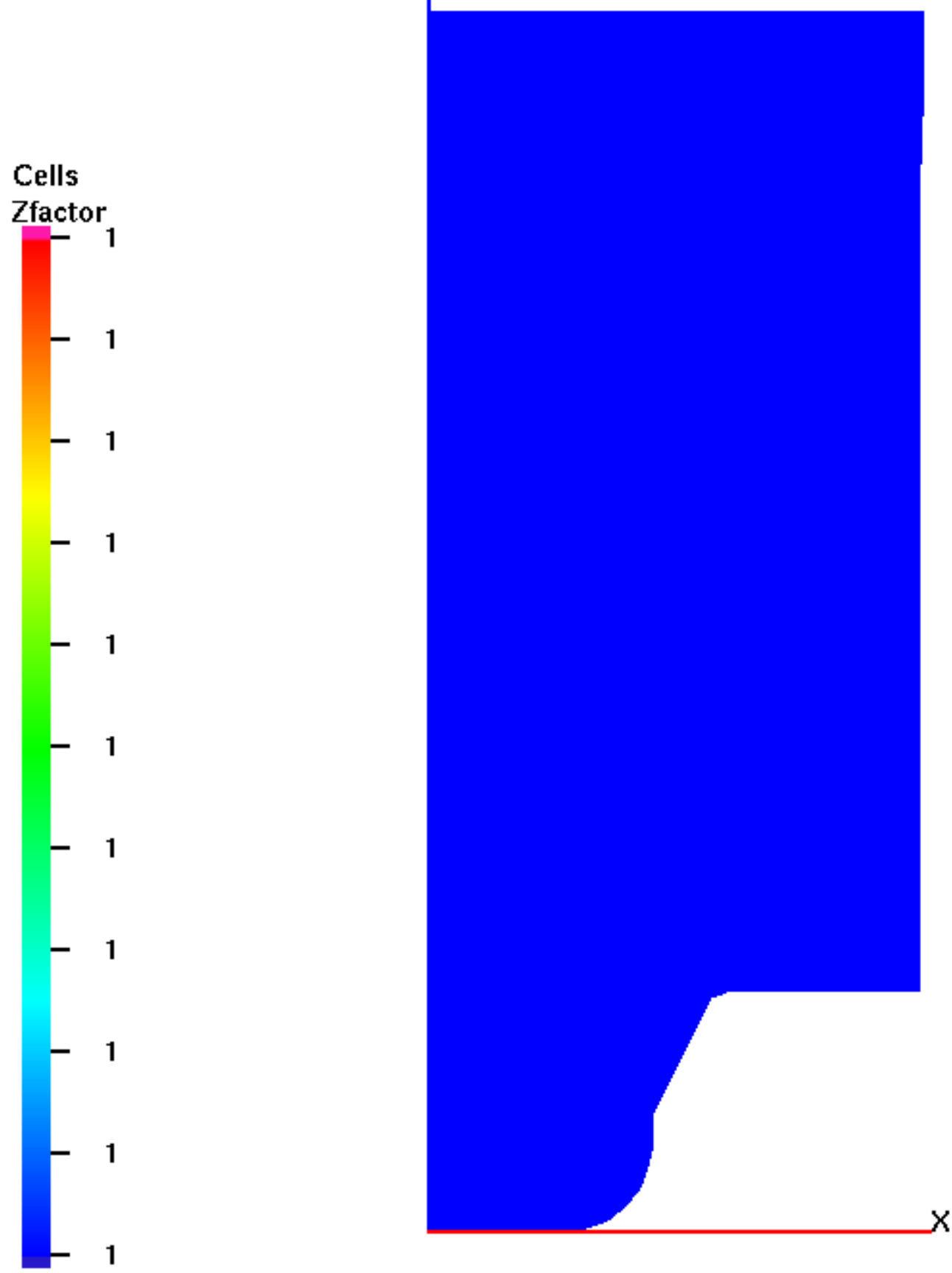

Figure 1: Z-factor for pure air at bottom center 


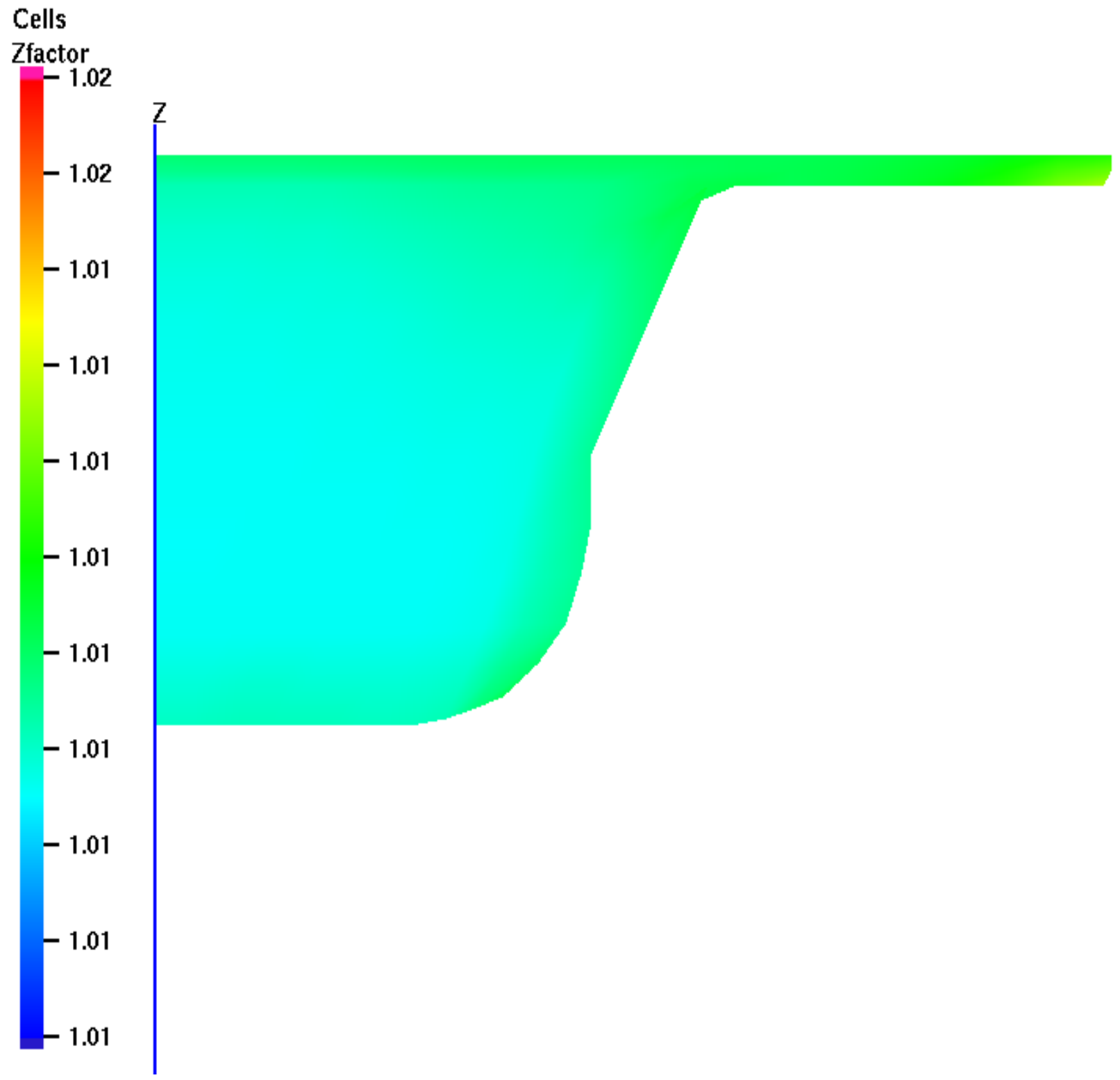

Figure 2: Z-factor for pure air at top center 

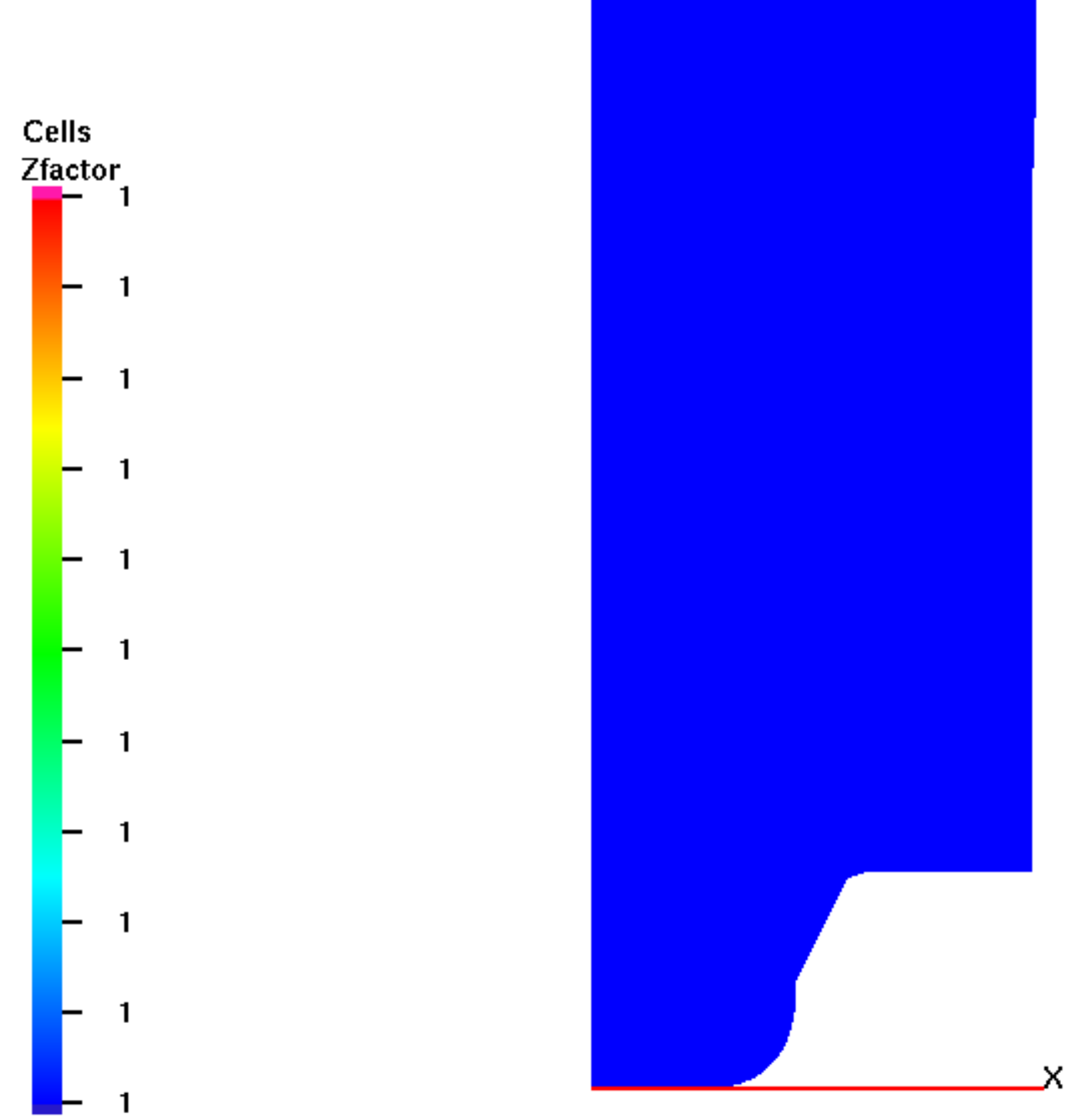

Figure 3: Z-factor for a mixture of $75 \% N_{2}$ and $25 \% C_{10} H_{8}$ at bottom center 


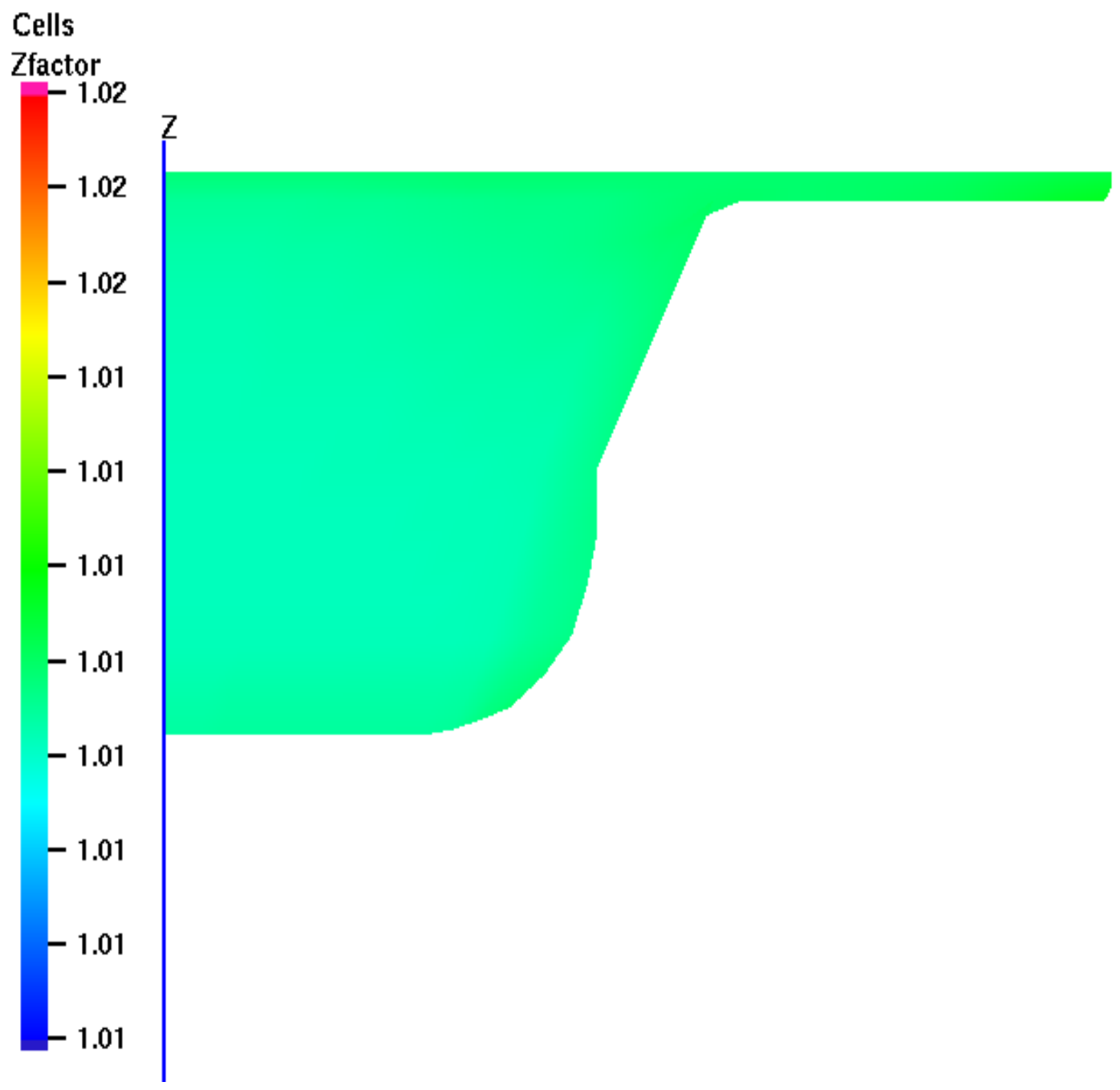

Figure 4: Z-factor for a mixture of $75 \% N_{2}$ and $25 \% C_{10} H_{8}$ at top center 
Cells

Zfactor

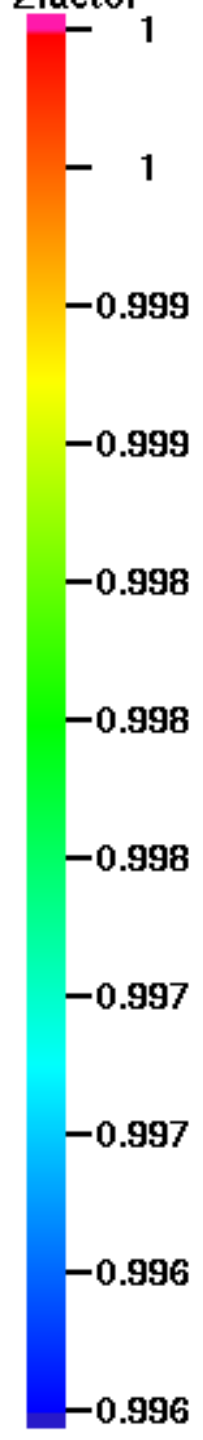

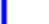

$3.156000 \mathrm{e}+00$

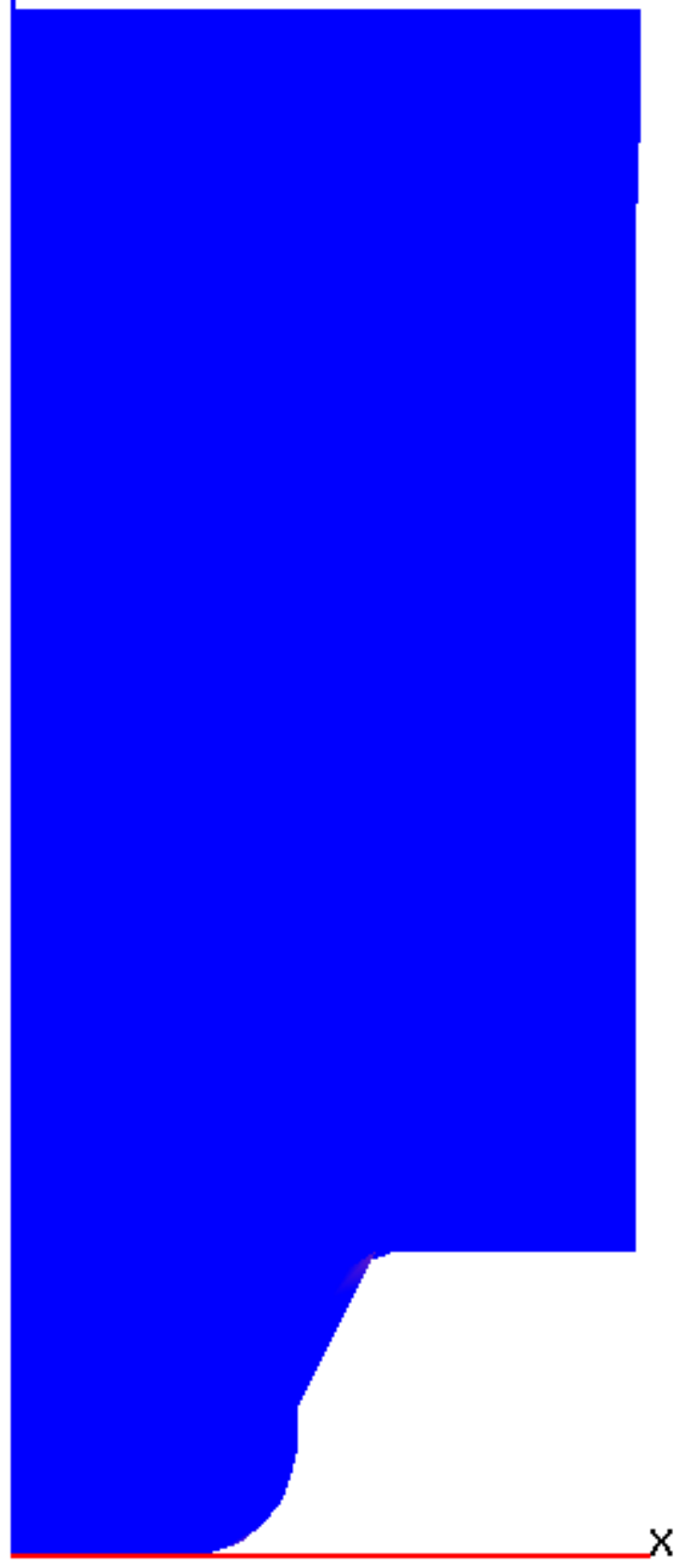

Figure 5: Z-factor for a mixture of $25 \% N_{2}$ and $75 \% C_{10} H_{8}$ at bottom center 


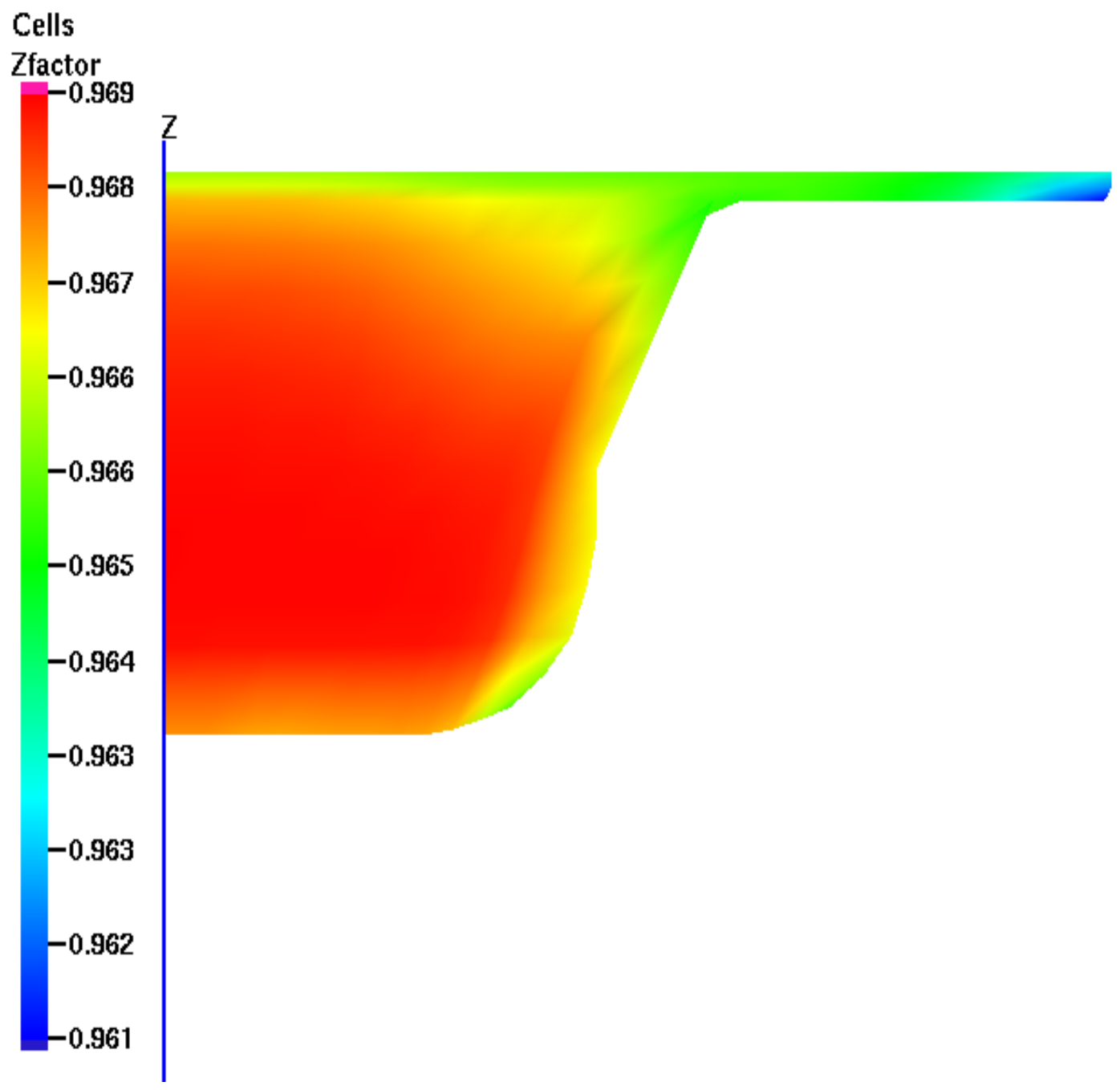

Figure 6: Z-factor for a mixture of $25 \% \mathrm{~N}_{2}$ and $75 \% \mathrm{C}_{10} \mathrm{H}_{8}$ at top center 

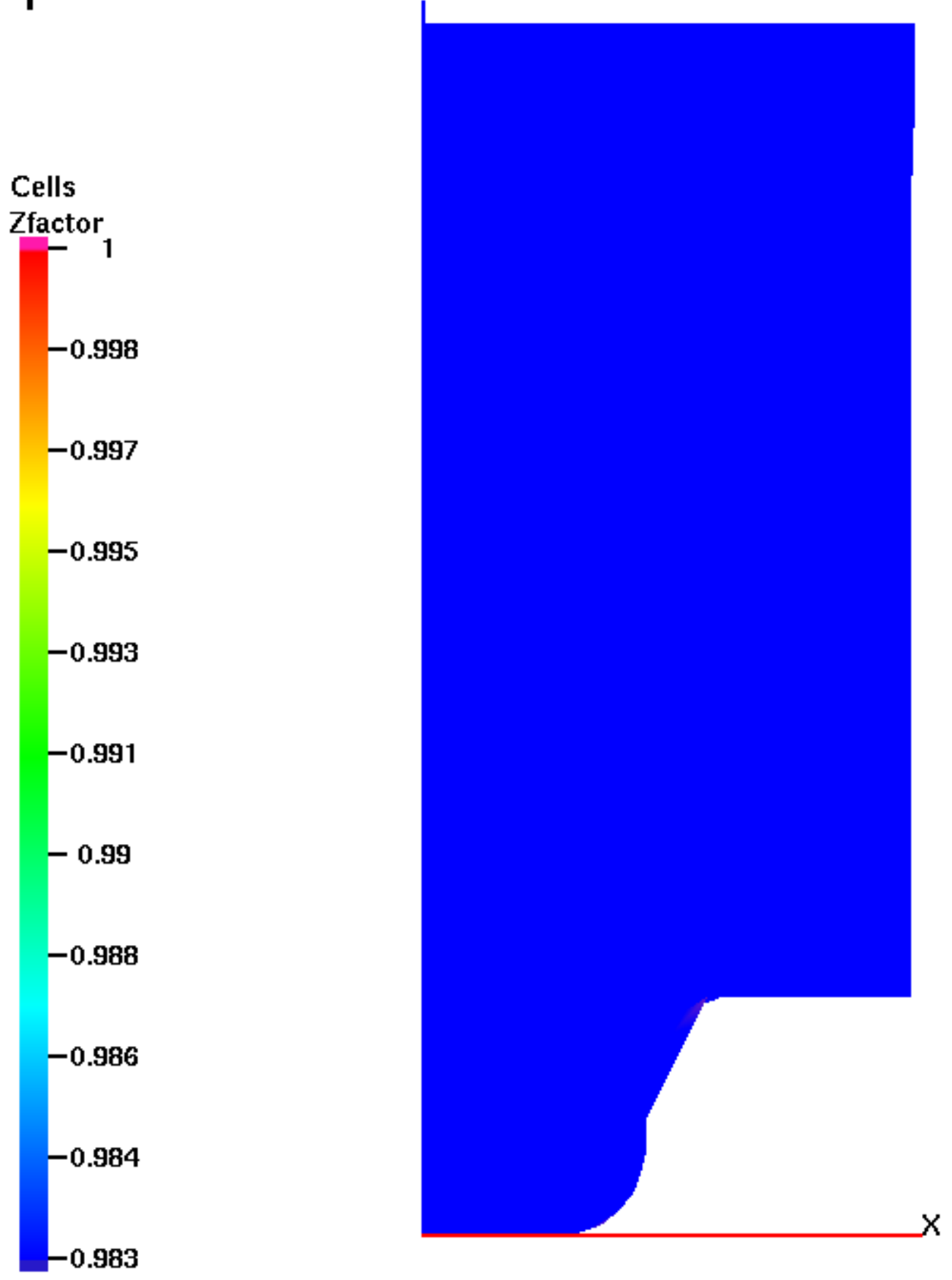

Figure 7: Z-factor for a mixture of $5 \% N_{2}$ and $95 \% C_{10} H_{8}$ at bottom center 


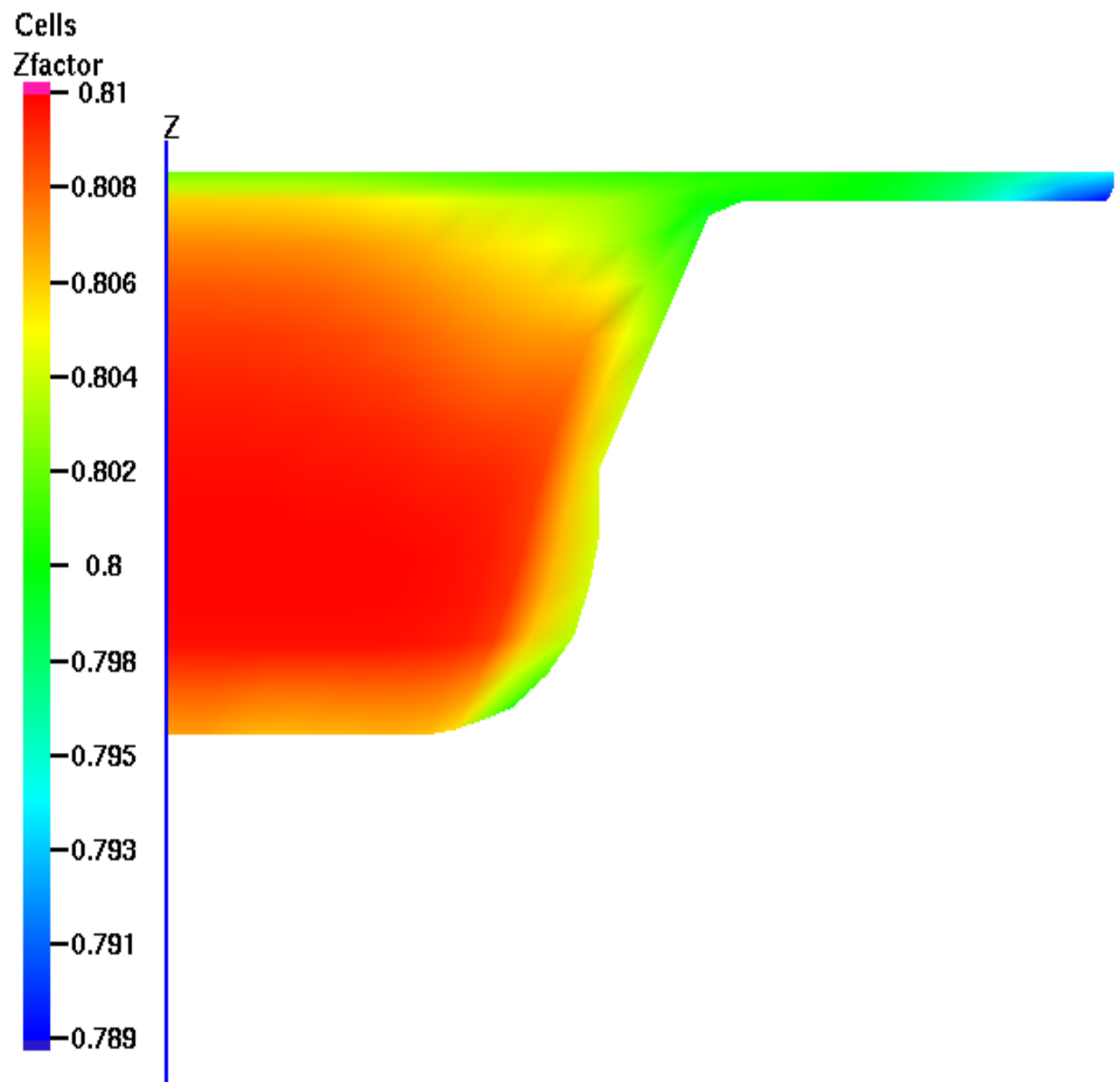

Figure 8: Z-factor for a mixture of $5 \% N_{2}$ and $95 \% C_{10} H_{8}$ at top center 


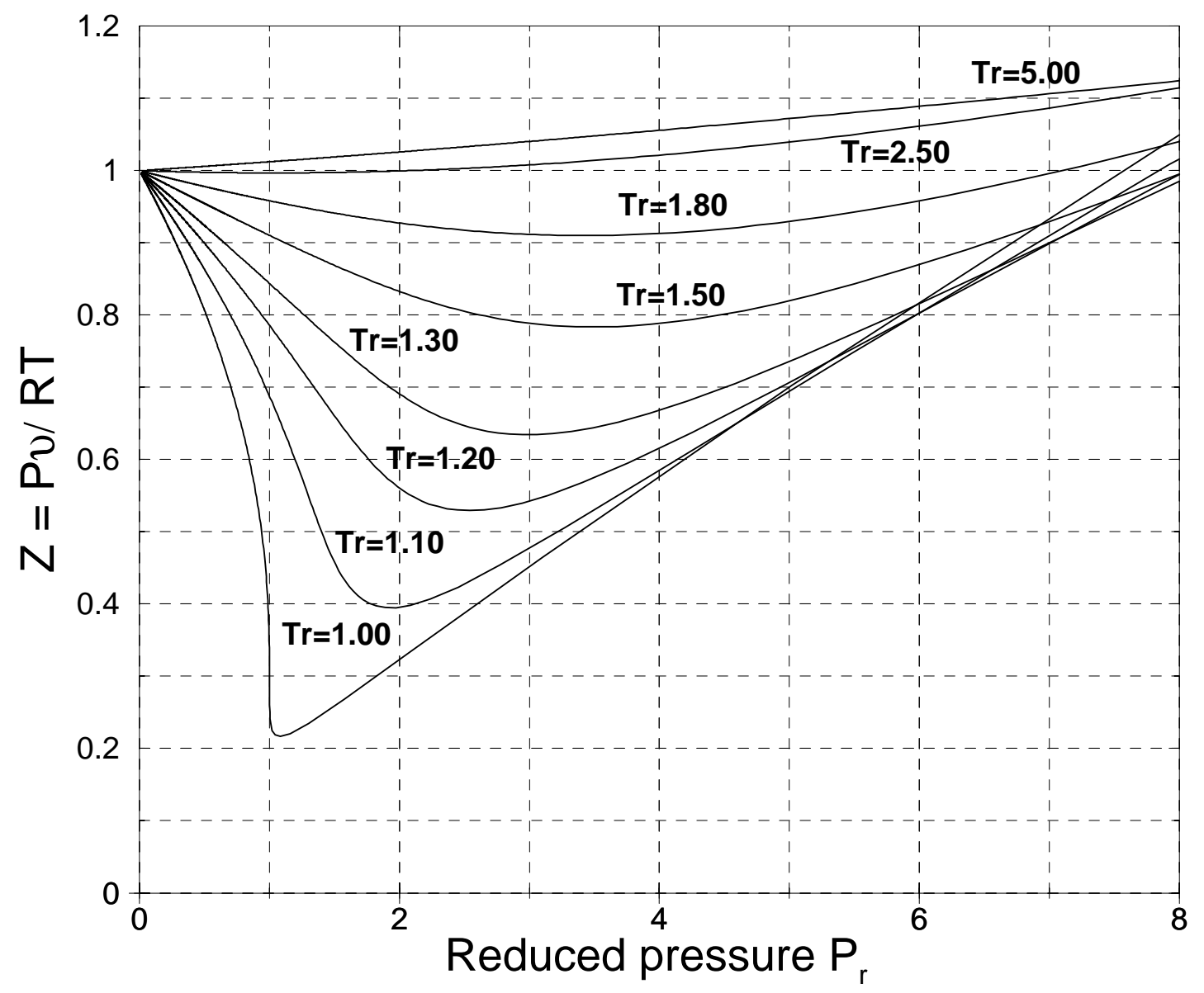

Figure 9: Compressibility chart for a simple fluid, calculated from the Lee Kesler modification of the BenedictWebb-Rubin equation of state [1]. 


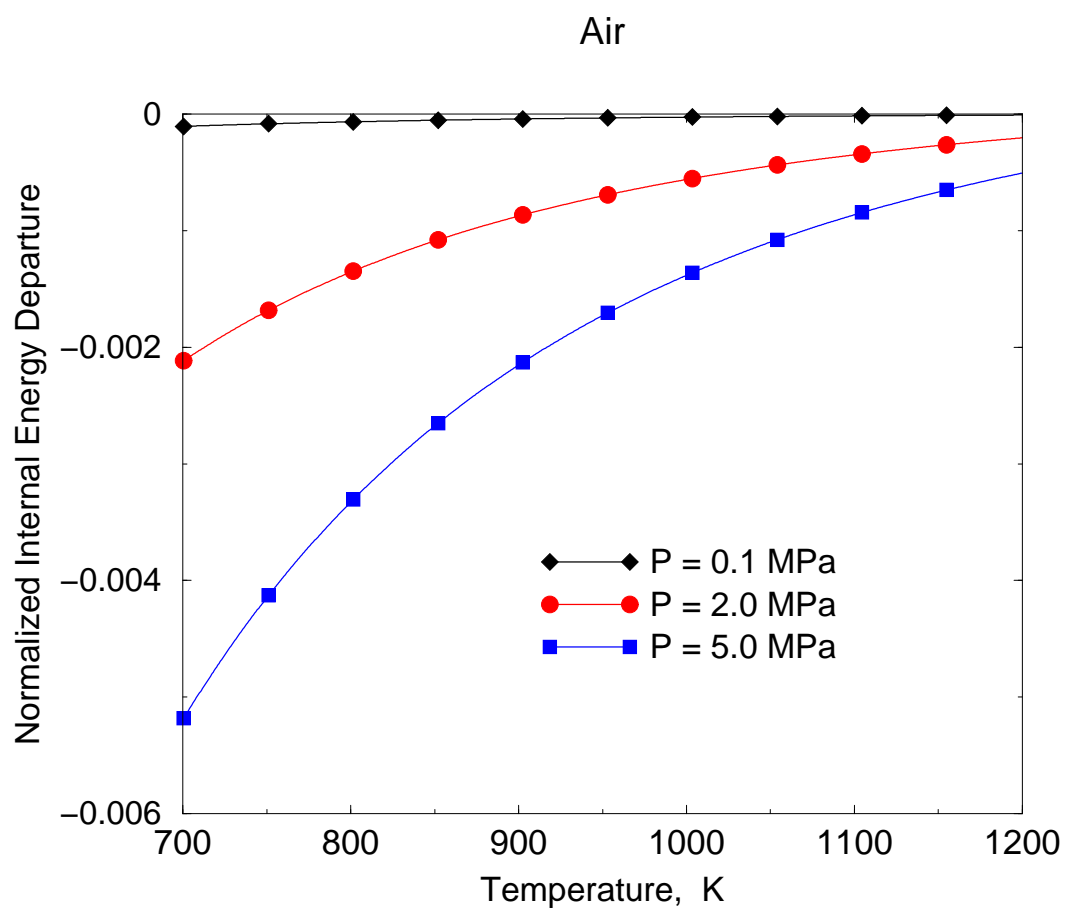

Figure 10: Normalized internal energy departure for air

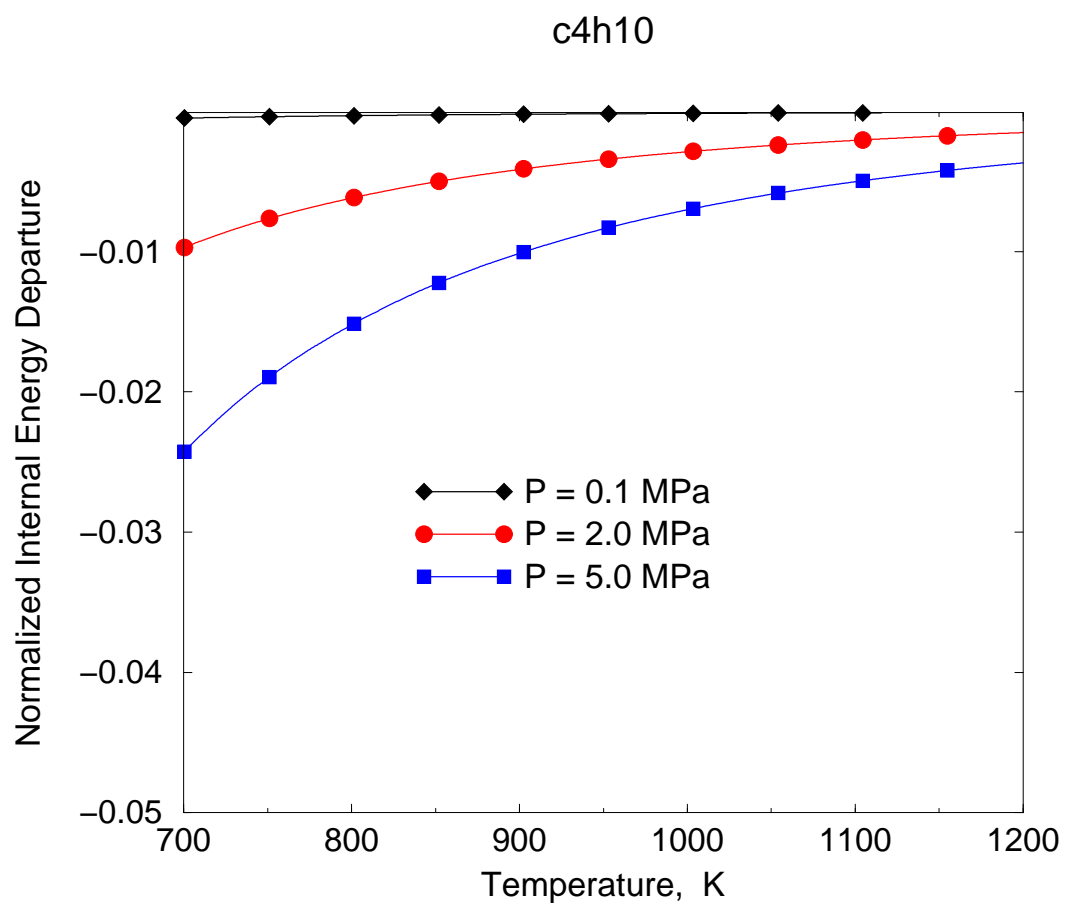

Figure 11: Normalized internal energy departure for $C_{4} H_{10}$. 


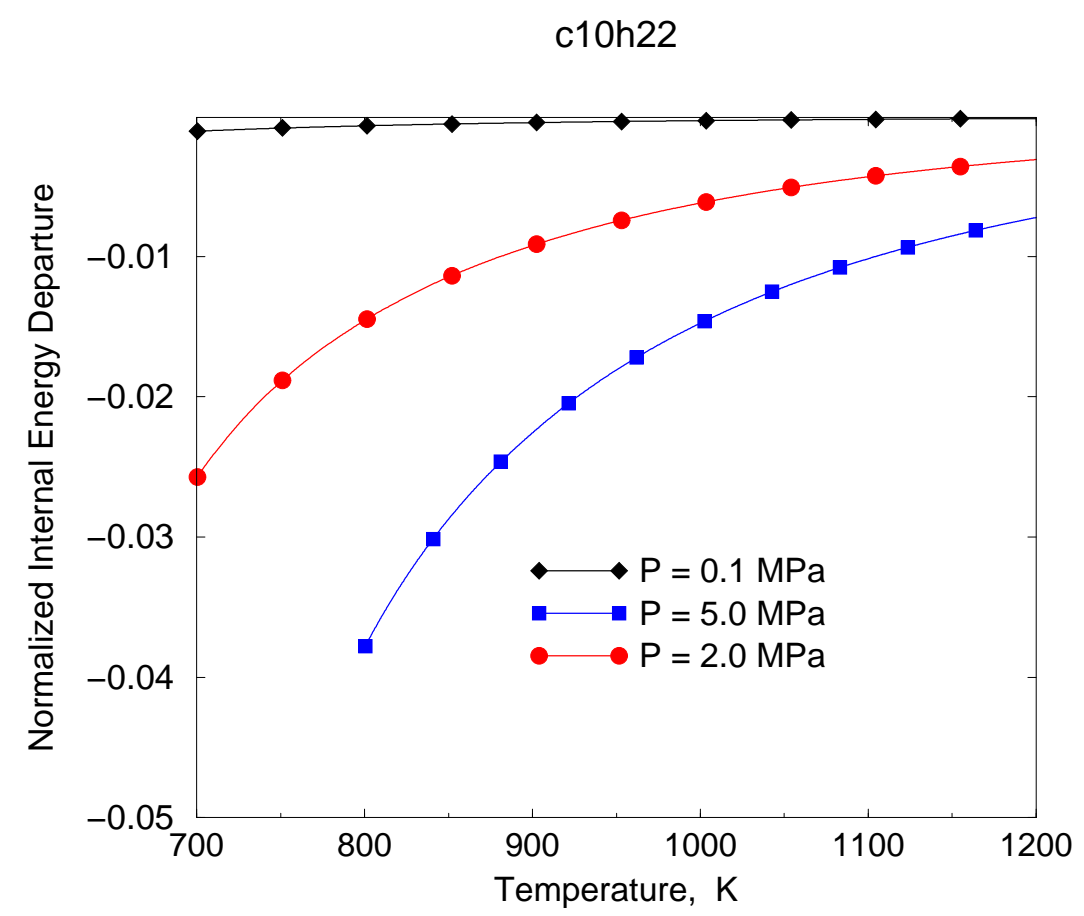

Figure 12: Normalized internal energy departure for $\mathrm{C}_{10} \mathrm{H}_{22}$.

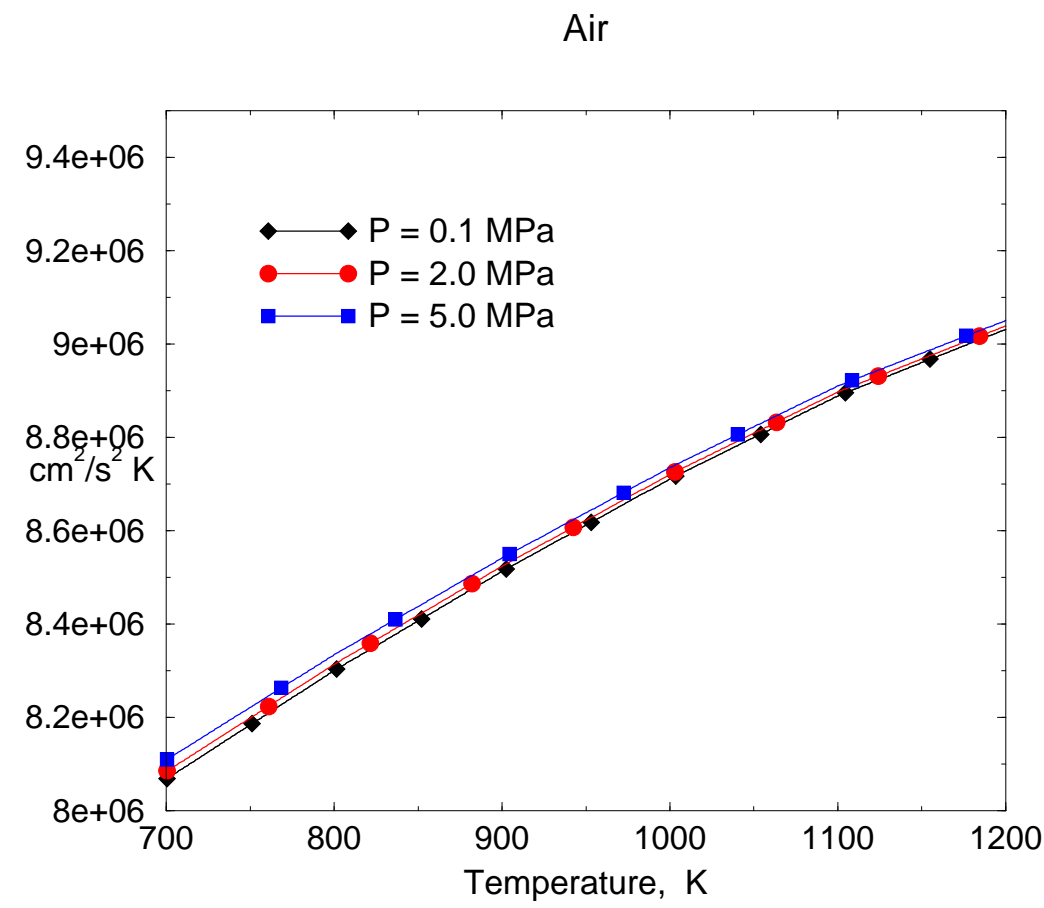

Figure 13: Pressure influence over constant volume specific heat behavior for air 
c4h10

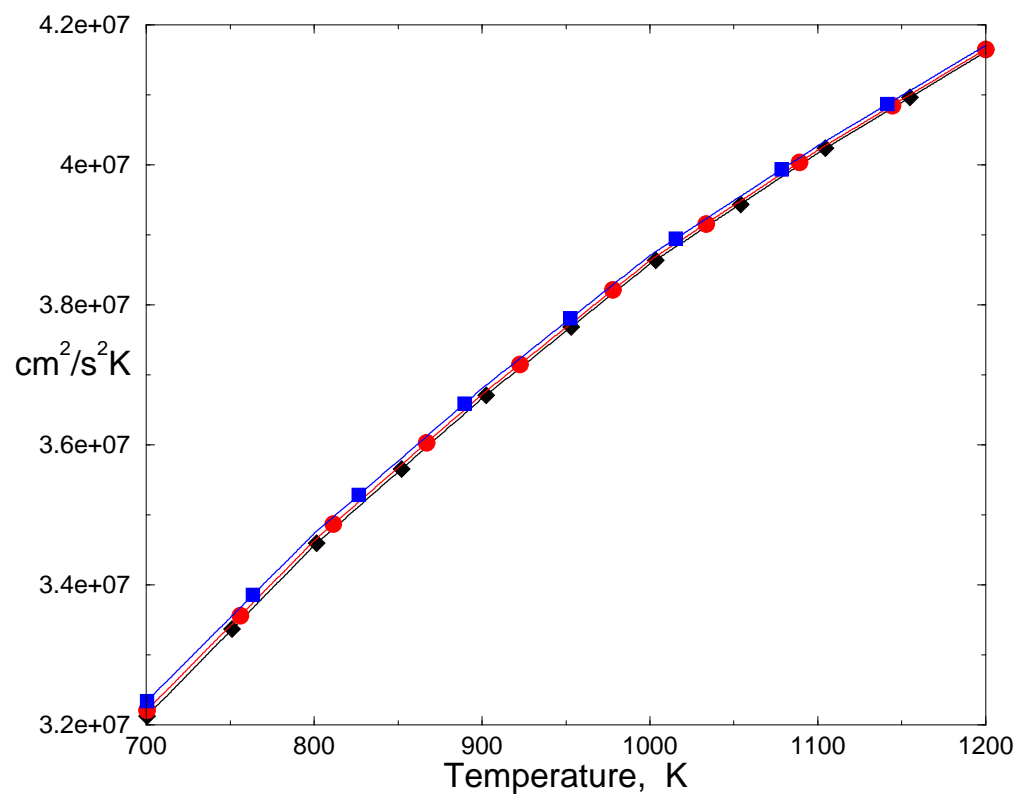

Figure 14: Pressure influence over constant volume specific heat behavior for $C_{4} H_{10}$.

\section{c10h22}

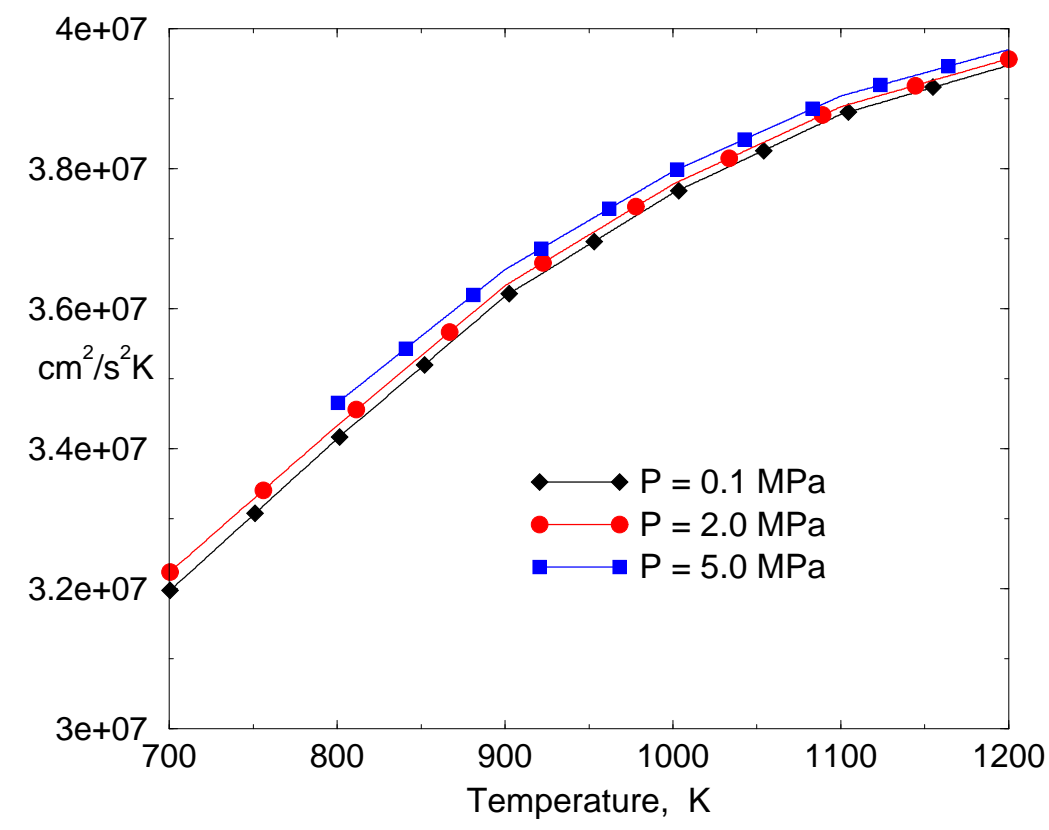

Figure 15: Pressure influence over constant volume specific heat behavior for $C_{10} H_{22}$. 
Air

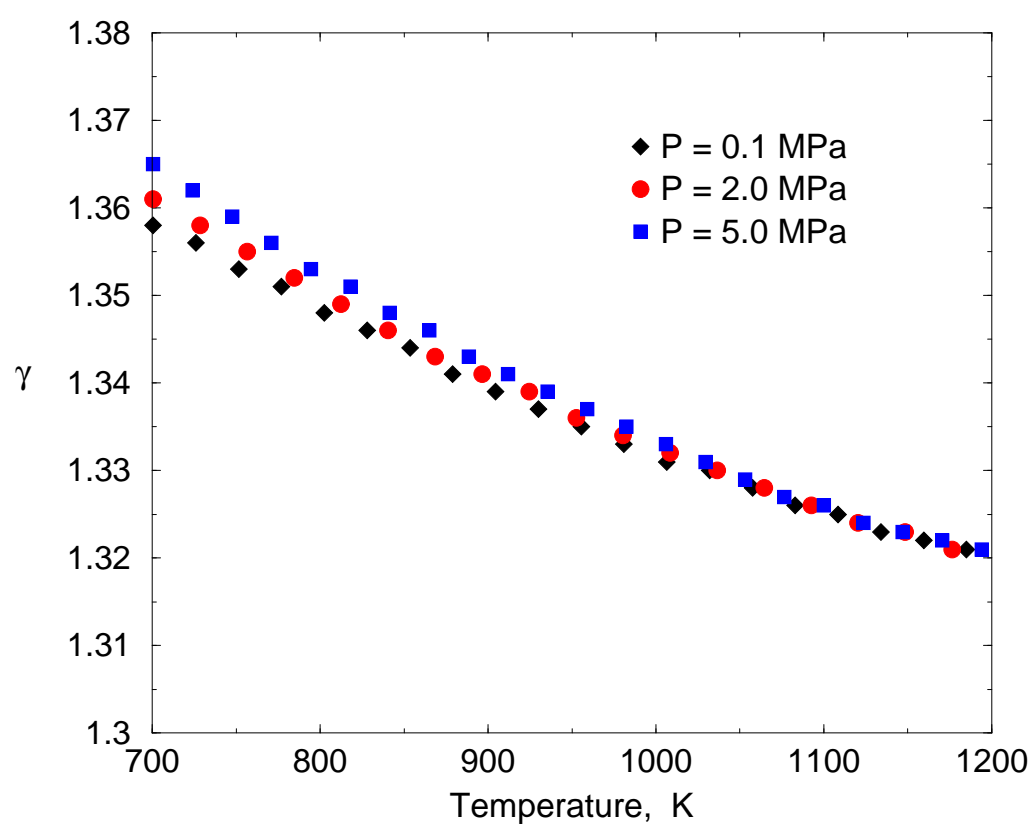

Figure 16: Specific heat ratio for air at various pressures

\section{c4h10}

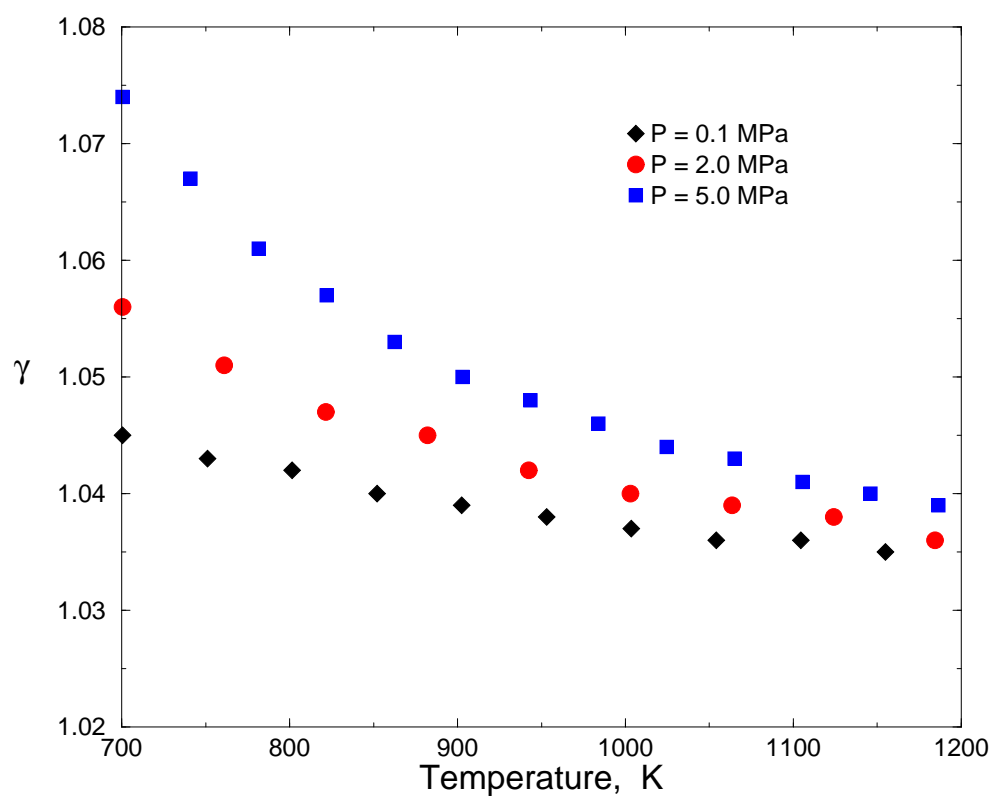

Figure 17: Specific heat ratio for $C_{4} H_{10}$ at various pressures 


\section{c10h22}

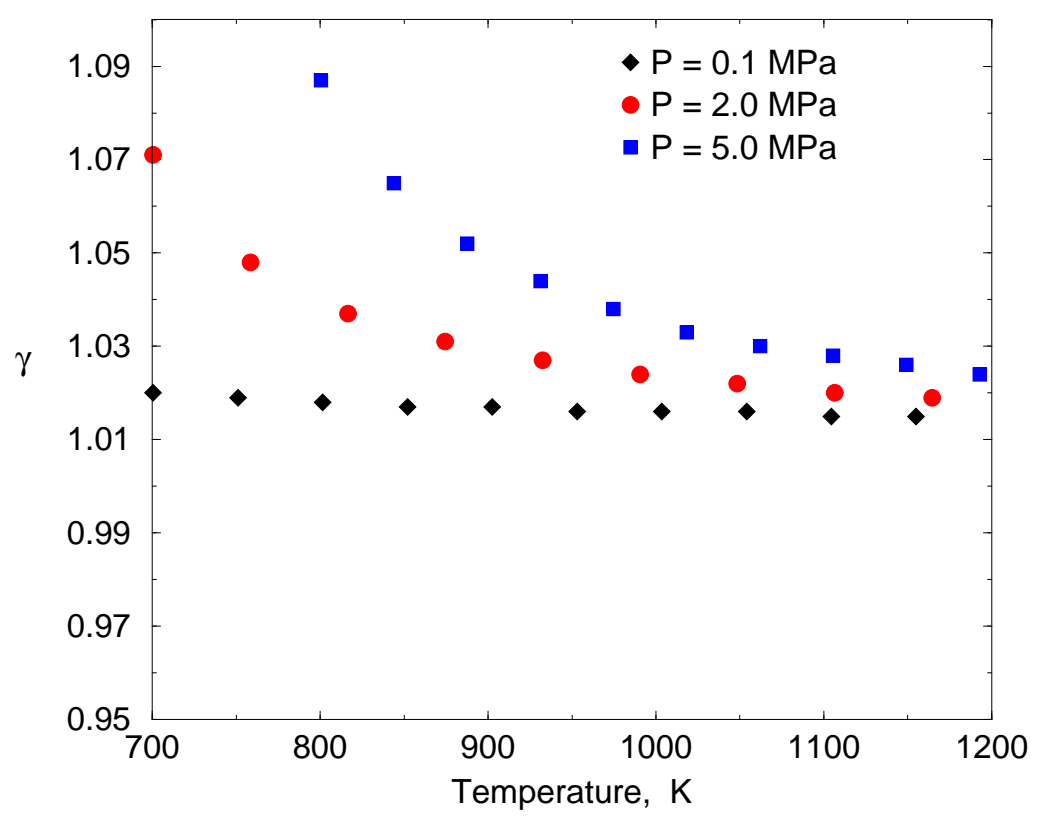

Figure 18: Specific heat ratio for $C_{10} H_{22}$ at various pressures

Air

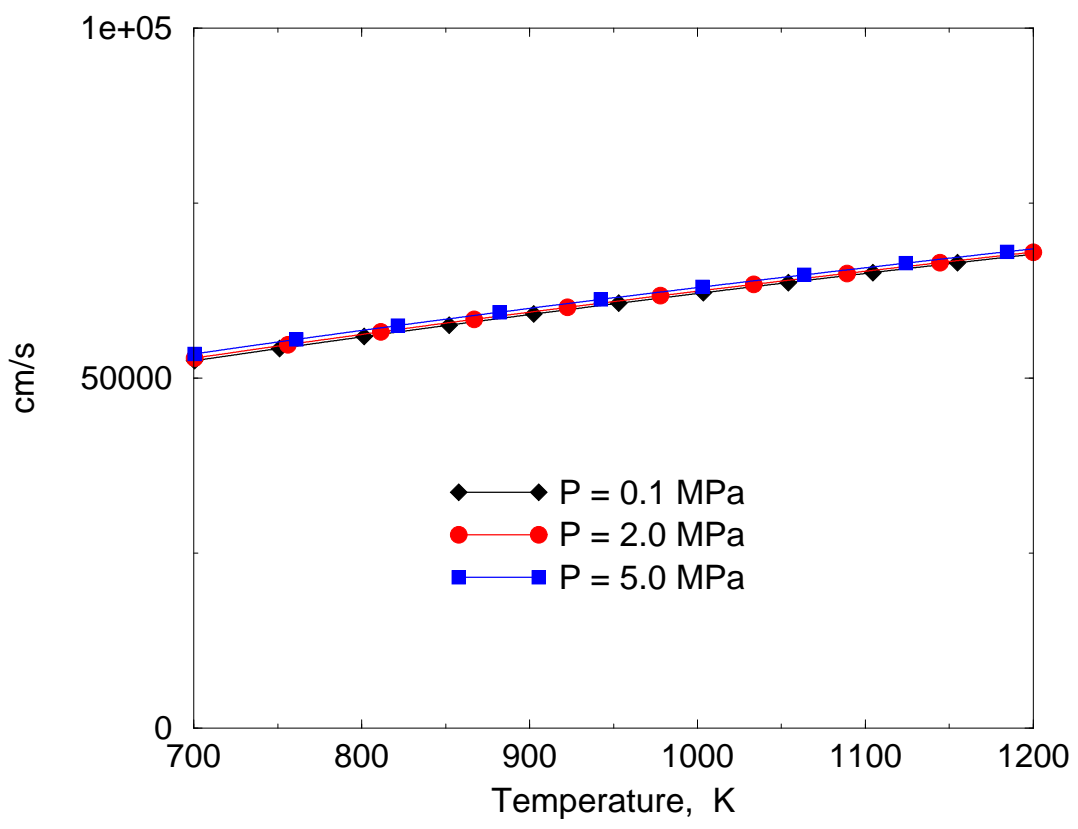

Figure 19: Isentropic sound speed for air. 


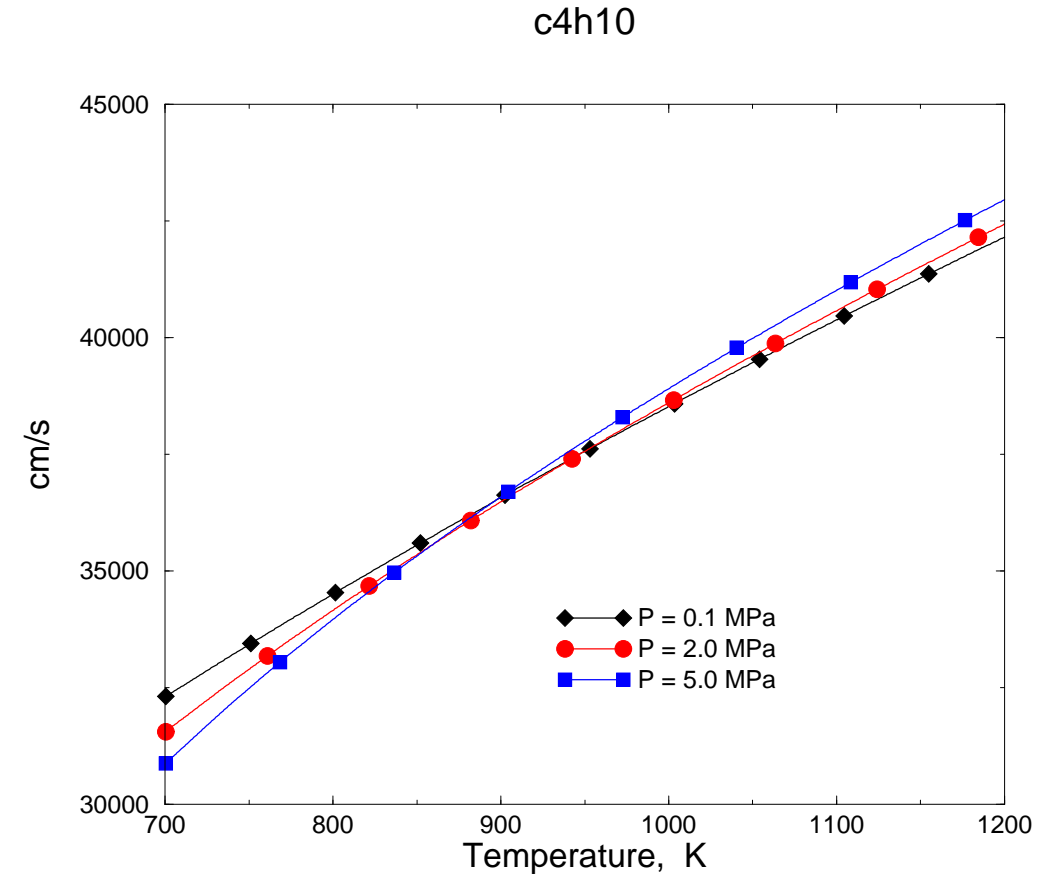

Figure 20: Isentropic sound speed for $C_{4} H_{10}$.

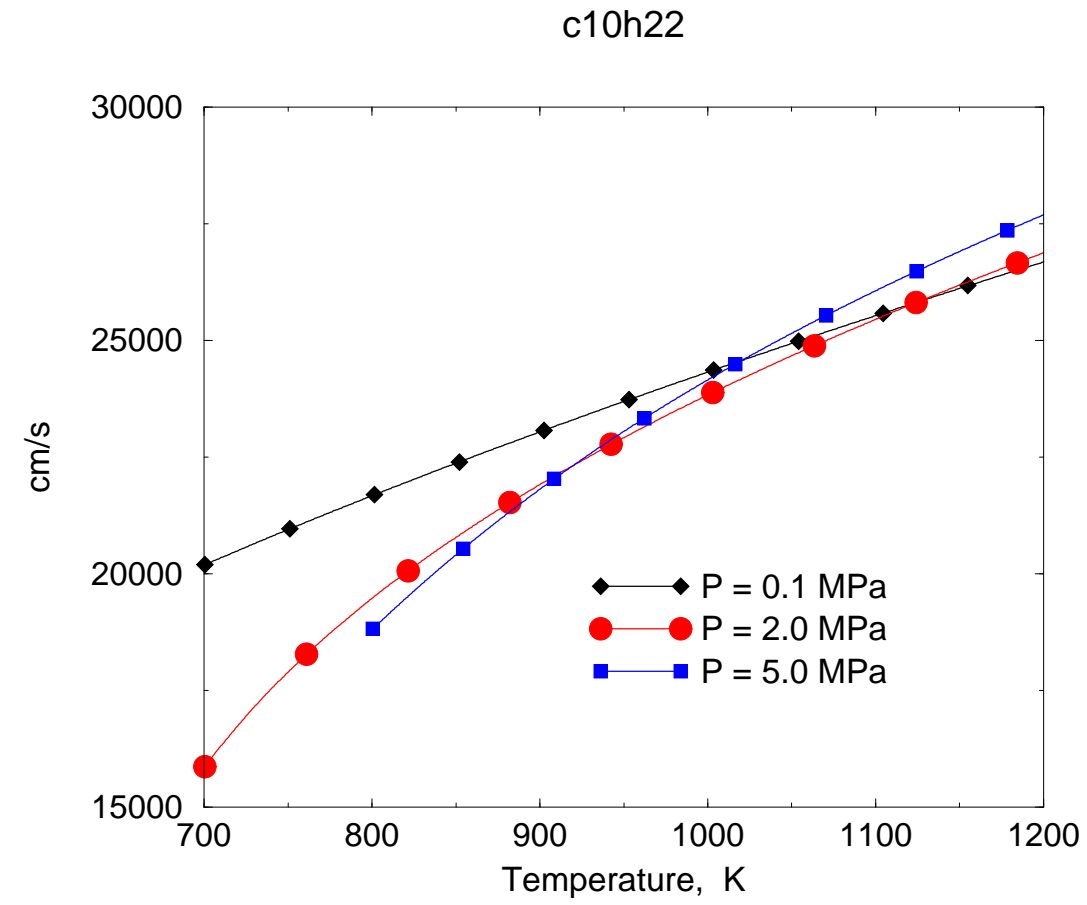

Figure 21: Isentropic sound speed for $C_{10} H_{22}$. 


\section{References}

[1] Robert C. Reid, John M. Prausnitz, and Bruce E. Poling. The Properties of Gases 85 Liquids, Fourth Edition. McGraw-Hill, Inc., New York, fourth edition, 1987.

[2] R.E. Sonntag and G.J. Van Wylen. Introduction to Thermodynamics Classical and Statistical. John Wiley \& Sons, New York, 1991.

[3] J.V. Sengers, R.F. Kayser, C.J. Peters, and H.J. White Jr. Equations of State for Fluids and Fluid Mixtures, volume V. Elsevier, Amsterdam, The Netherlands, 2000.

[4] D.Y. Peng D.B. Robinson. A new two-constant equation of state. Ind. Eng. Chem. Fundam., 15(1), 1976.

[5] G.S. Zhu and S.K. Aggarwal. Transient supercritical droplet evaporation with emphasis on the effects of equation of state. International Journal of Heat and Mass Transfer, 43, 2000.

[6] H. Knapp. Vapor-liquid Equilibria for Mixtures of Low-Boiling Substances, volume 6. DECHEMA, Frankfurt, Germany, 1982.

[7] Ohe S. Vapor-Liquid Equilibrium at High Pressure. Elsevier, 1990.

[8] H. Jia and G. Gogos. High pressure droplet vaporization; effects of liquid-phase gas solubility. International Journal of Heat and Mass Transfer, 36:4419-4431, 1993. 


\section{A Notation}

$C_{p}=$ Constant pressure specific heat $C_{v}=$ Constant volume specific heat

$C_{s}=$ Isentropic sound speed

$C_{T}=$ Isothermal sound speed

$\gamma=$ Specific heat ratio $C_{p} / C_{v}$

$H, h=$ Enthalpy per unit mass

$I=$ Internal energy per unit mass

$M=$ Mass

$P=$ Pressure

$R_{u}$ Universal gas constant

$\rho=$ Density

$S=$ Entropy per unit mass

$T=$ Temperature

$V=$ Volume

$v=$ Specific volume $\left(L^{3} / M\right)$

$\bar{v}=$ Molar specific volume $\left(L^{3} / M_{o}\right)$

$Y_{m}, y_{m}=$ Mass fraction of species $\mathrm{m}$

$X_{m}, x_{m}=$ Mole fraction of species $\mathrm{m}$

$Z=$ Compressibility factor 


\section{B Changes to the Code}

A sizable number of changes were made to KIVA-3V in order to generalize the thermodynamic relations from ideal behavior to real-gas behavior. A list detailing these changes is included below.

\section{BCCCIN.f}

- Change from

$$
T E M P A M B=\frac{P(i 4)}{R G A S} \frac{1}{\sum_{m} S P D_{m} / \omega_{m}}
$$

to

$$
\text { tempamb }=\operatorname{eos}(p(i 4), 1 / x x, 0.0,3),
$$

where $x x=\sum_{m} S P D_{m} / \omega_{m}$, or simply

$$
\text { tempamb }=p(i 4) /(x x * \text { rgas })
$$

in the case of ideal gas conditions.

\section{CHEMEQ.f}

- Change from

$$
C P T=\frac{G A M M A(i 4) * P(i 4)}{R O(i 4) \times T E M P(i 4) \times(G A M M A(i 4)-1.0)} \Longrightarrow \frac{\gamma R}{\gamma-1}
$$

to either

$$
c p t=\operatorname{cvheat}(\ldots)+\frac{\operatorname{temp}(i 4)}{\operatorname{ro}(i 4)^{2}} \frac{\operatorname{dpdtro}(\ldots)^{2}}{\operatorname{dpdrot}(\ldots)}
$$

for general EOS, where the partial derivatives are computed based on PR. The other option is to use the original calculation above.

\section{CHMQGM.f}

- Change from

$$
C P T=\frac{G A M M A(i 4) * P(i 4)}{R O(i 4) \times T E M P(i 4) \times(G A M M A(i 4)-1.0)} \Longrightarrow \frac{\gamma R}{\gamma-1}
$$

to either

$$
c p t=\operatorname{cvheat}(\ldots)+\frac{\operatorname{temp}(i 4)}{\operatorname{ro}(i 4)^{2}} \frac{\operatorname{dpdtro}(\ldots)^{2}}{\operatorname{dpdrot}(\ldots)}
$$

for general EOS, where the partial derivatives are computed based on PR.The other option is to use the original calculation above.

\section{DRDP.f}

- Change from

$$
R D R D P=1.0 /\left[-\frac{V O L B(I 4)}{\gamma P(I 4)}-\Delta T * P H I P * R P G S 2 *(S I P+\ldots)\right]
$$

to

$$
R D R D P=1.0 /[D V D P S-\Delta T * P H I P * R P G S 2 *(S I P+\ldots)] .
$$




\section{DRDT.f}

- Here, we compute the derivative of the residual $R E S(i 4)$ with respect to $T^{B}$ and take the inverse.

$$
\begin{aligned}
& \frac{d R E S(i 4)}{d T^{B}}=1-\frac{C V T E R M * \Delta t K(\text { geo }) \phi_{D}}{\left.M \frac{\partial I}{\partial T}\right|_{P}}, \\
& R D R D T(i 4)=\left[\frac{d R E S(i 4)}{d T^{B}}\right]^{-1}=\left[1-\frac{C V T E R M * R P R * S U M T}{\left.M * \frac{\partial I}{\partial T}\right|_{P}}\right]^{-1} .
\end{aligned}
$$

This replaces the original,

$$
R D R D T(I 4)=\left[1-\frac{C V T E R M(I 4) * R P R * S U M T}{R O(I 4) * V O L(I 4) * C V(I 4)}\right]^{-1} .
$$

\section{EXDIF.f}

- Change from

$$
C P C(i 4)=\frac{G A M M A(i 4) \times P(i 4)}{(G A M M A(i 4)-1.0) \times R O N(i 4) \times T E M P(i 4)}
$$

to

$$
\operatorname{cpc}(i 4)=\operatorname{cvheat}(\ldots)+\frac{\operatorname{temp}(i 4)}{\operatorname{ron}(i 4)^{2}} \frac{d p d \operatorname{tro}(\ldots)^{2}}{d p d r o t(\ldots)}
$$

for general EOS, where the partial derivatives are computed based on PR. For the ideal-gas case the original version is used.

\section{LAWALL.f}

- Change from

$$
C P=\frac{G A M M A(i 4) \times P(i 4)}{(G A M M A(i 4)-1.0) \times R O(i 4) \times T E M P(i 4)}
$$

to

$$
c p=\operatorname{cvheat}(\ldots)+\frac{\operatorname{temp}(i 4)}{\operatorname{ro}(i 4)^{2}} \frac{\operatorname{dpdtro}(\ldots)^{2}}{\operatorname{dpdrot}(\ldots)}
$$

for general EOS, where the partial derivatives are computed based on PR. For the ideal-gas case the original version is used.

\section{PHASEB.f}

- Change from

$$
\operatorname{VOLLTE}=\operatorname{VOLB}(i 4) *(1.0+R G A M M A(i 4) *[1.0-P(i 4) * R P A(i 4)])
$$

( This is based on

$$
\left.\frac{V_{t e}-V^{B}}{V^{B}}=\frac{1}{\gamma} \frac{P_{a}-P}{P_{a}} \Longrightarrow \frac{d V}{V}=\frac{1}{\gamma} \frac{d P}{P} \Longrightarrow \frac{V_{2}}{V_{1}}=\left(\frac{P_{1}}{P_{2}}\right)^{1 / \gamma} .\right)
$$

to

$$
V O L L T E=\operatorname{VOLB}(i 4)+D V D P S(i 4) *(P(i 4)-P P R E D(i 4)) .
$$




\section{PINIT.f}

- Original

$$
P H I P T E=D T * S Q R T(G A M M A(i 4) * R P G S 2 * P(i 4) / R O(i 4) * R D 138)
$$

replaced by

$$
\text { PHIPTE }=D T * S Q R T\left(C_{s}^{2} * R P G S 2 * R D 138\right),
$$

where $C_{s}^{2}$ is calculated for either an ideal or PR equation of state following Eq. (17).

\section{PSOLVE.f}

- Omit $R P A(i 4)=1.0 / P(i 4)$ in favor of

$$
\operatorname{PPRED}(i 4)=P(i 4) \text {. }
$$

- Toward the end of the subroutine, delete PPRED =1.0/RPA(i4). PPRED(i4) has already been defined.

- Related to this, replace

$$
P E R R=M A X(P E R R, A B S(P(I 4)-P P R E D) * R P D I F)
$$

with

$$
P E R R=M A X(P E R R, A B S(P(I 4)-P P R E D(i 4)) * R P D I F) .
$$

\section{RESP.f}

- Eq. (112) of the KIVA-II manual

$$
V O L P=\operatorname{VOLB}(I 4) *\left[1.0+\frac{1}{\gamma}(1.0-P(I 4) * R P A(I 4))\right]
$$

is changed to

$$
V O L P=V O L B(I 4)+D V D P S *(P(i 4)-P P R E D(i 4)) .
$$

\section{REST.f}

- We want to calculate the residual of Eq. (28). This is done by subtracting the r.h.s of Eq. (28) from the l.h.s. The implicit part of the heat diffusion is also subtracted. The original version is

$$
\begin{array}{r}
R E S(i 4)=\operatorname{TEMP}(i 4)+C V T E R M\left[\frac{S I E(i 4)-S I E T I L(i 4)}{C v(i 4)}-\operatorname{TTIL}(i 4)\right. \\
\left.-\frac{\Delta t\left(K \nabla T^{B} \cdot \mathbf{A}_{\mathbf{a}} \phi_{D}\right)}{C v(i 4) * R O(i 4) * V O L(i 4)}\right]
\end{array}
$$

and is replaced by

$$
\begin{array}{r}
R E S(i 4)=T E M P(i 4)-C V T E R M\left[\frac{\operatorname{SIETIL}(i 4)-S I E(i 4)}{\left.\frac{\partial I}{\partial T}\right|_{P}}+\operatorname{TTIL}(i 4)\right. \\
\left.+\frac{\Delta t\left(\sum_{\alpha} K_{\alpha}^{n} \nabla\left(\phi_{D} T^{B}\right)_{\alpha} \cdot \mathbf{A}_{\alpha}^{\mathbf{n}}\right.}{\left.R O(i 4) * V O L(i 4) * \frac{\partial I}{\partial T}\right|_{P}}\right] .
\end{array}
$$




\section{SETUP.f}

- Change from

$$
T E M P A M B=\frac{P A M B}{\left(\sum_{m} S P D A M B_{m} \times R M W_{m}\right) R_{u}}
$$

to

$$
\text { tempamb }=\operatorname{eos}(\text { pamb, } 1 / x x, 0.0,3)
$$

or

$$
\text { tempamb }=p a m b /(x x * r g a s),
$$

depending on whether we apply the ideal or PR EOS. $f m o l$ array is computed previously and $x x$ is total molar density.

- Change from

$$
T E M P I N=P C C E F F /\left(\sum_{m} S P D I N 0_{m} \times R M W_{m}\right) R_{u}
$$

to

$$
\text { tempin }=\operatorname{eos}(\text { pccef } f, 1 / x x, 0.0,3)
$$

or

$$
\text { tempin }=\text { pcceff } /(x x * \text { rgas }) \text {, }
$$

depending on whether we apply the ideal or PR EOS. $f m o l$ array is computed previously and $x x$ is total molar density.

$$
G A M M A(i 4)=1.4 * f a c
$$

when $i 4$ is an obstacle or a ghost cell.

\section{SETUPRO.f}

- Change from

$$
S U M R O=\frac{\operatorname{PRESI}(n)}{W T O T D E N \times R_{u} T E M P I(n)}=\frac{P R E S I(n)}{\left(\sum_{m} Y_{m} / \omega_{m}\right) R_{u} T}
$$

to

$$
\begin{gathered}
\text { vmolmix }=\operatorname{eos}(\operatorname{presi}(n), 0.0, \text { tempi }(n), 2), \\
\text { sumro }=\frac{1.0}{\text { vmolmix } * \text { wtotden }},
\end{gathered}
$$

or

$$
\text { sumro }=\operatorname{presi}(n) /(\text { wtotden } * \text { rgas } * \text { tempi }(n)) \text {, }
$$

depending on whether we apply ideal or PR EOS. 
- Change from

$$
P C H K=S U M R O O W * T E M P I(n) * R_{u} \Longrightarrow P=\sum_{m} \frac{\rho_{m}}{\omega_{m}} R_{u} T
$$

to

$$
\text { pchk }=\operatorname{eos}(0.0,(1.0 / \text { sumroow }), \text { tempi }(n), 1)
$$

or

$$
\text { pchk }=\text { sumroow } * \text { tempi }(n) * \text { rgas },
$$

depending on which EOS is used.

\section{STATE.f}

\section{Major changes made to this subroutine}

- Change from

$$
p(i 4)=\left(\sum_{i} S P D_{i} * R M W_{i}\right) * R G A S * T E M P(i 4)
$$

to

$$
p(i 4)=\operatorname{eos}(0.0,(1.0 / p(i 4)), \operatorname{temp}(i 4), 1)
$$

or

$$
p(i 4)=p(i 4) * \text { rgas } * \operatorname{tem} p(i 4),
$$

depending on which EOS is employed.

- The calculation for internal energy departure handled by function DELTAE.f is introduced.

$$
C S U B V=(E H I-E L O) \times 0.01
$$

is left unchanged, since by definition, the constant-volume specific heat is the same regardless of which EOS is employed.

$$
G A M M A(i 4)=1.0+\frac{P(i 4)}{R O(i 4) \times C S U B V \times T E M P(i 4)}
$$

\section{TINVRT.f}

\section{Major changes made to this subroutine}

- The inclusion of $D E L T A E()$ to calculate internal energy departure in both computations of $E L O$ and EHI;

- The calculation of $C_{v}$ based on either ideal or PR-EOS;

- Calculation of $\left.\frac{\partial I}{\partial T}\right|_{P}$.

$$
G A M M A(I 4)=\frac{C V(I 4)+R(I 4)}{C V(I 4)} .
$$




\section{TSOLVE.f}

\section{Major changes made to this subroutine}

- The volume at the t-state, $V^{t}$, is calculated either for the ideal gas case, which is the original formulation, or the PR case.

- $\left.\frac{\partial V}{\partial T}\right|_{P}$ is calculated since it appears now in the new and more general energy equation. It is computed for either the ideal or PR case. Note that this derivative is computed at the t-state. In the PR case, the additional derivatives $\left.\frac{\partial P}{\partial T}\right|_{\rho}$ and $\left.\frac{\partial P}{\partial \rho}\right|_{T}$ are also computed.

- CVTERM is the denominator in the temperature equation (28) or (29). It is given originally by

$$
\text { CVTERM }=\left[1+\frac{P^{n}+P^{B}}{2 C_{v} P(i 4)} \bar{R}\right]^{-1},
$$

where $\bar{R}$ is the averaged gas constant. This is replaced by

$$
C V T E R M=\left[1+\left.\left(\frac{P^{n}+P^{B}}{\left.2 M^{B} \frac{\partial I}{\partial T}\right|_{P}}\right) \frac{\partial V}{\partial T}\right|_{P}\right]^{-1} .
$$

- SIETIL(i4)-SIE(i4) originally from the numerator of Eq. (29),

$$
\left(\frac{P^{n}+P^{B}}{2 \rho}\right)+\frac{\Delta t}{M^{B}}\left(K\left(1-\phi_{D}\right) \nabla \tilde{T} \cdot \mathbf{A}_{\mathbf{a}}+\left(1-A_{o}\right) \mathcal{D}_{i s s}\right),
$$

is replaced by

$$
\left(\frac{P^{n}+P^{B}}{2 M^{B}}\right)\left[\left.\frac{\partial V}{\partial T}\right|_{P} T^{t}-\left(V^{t}-V^{n}\right)\right]+\frac{\Delta t}{M^{B}}\left(K_{\alpha}^{n} \nabla\left[\left(1-\phi_{D}\right) \tilde{T}\right]_{\alpha} \cdot \mathbf{A}_{\alpha}^{\mathbf{n}}+\left(1-A_{o}\right) \mathcal{D}_{i s s}\right) .
$$

- $T^{B}$, originally given in the code as

$$
T^{B}=C V T E R M\left[T^{t}+\frac{\operatorname{SIETIL}(i 4)-\operatorname{SIE}(i 4)}{C_{v}}\right],
$$

is replaced by

$$
T^{B}=C V T E R M\left[T^{t}+\frac{\operatorname{SIETIL}(i 4)-S I E(i 4)}{\left.\frac{\partial I}{\partial T}\right|_{P}}\right] .
$$

- Once the temperature $T^{B}$ has been calculated, the calculation for volume quickly follows in the original code using the ideal gas law, i.e.,

$$
\operatorname{VOLB}(I 4)=R O(I 4) * \operatorname{VOL}(I 4) * R(I 4) * T E M P(I 4) / P(I 4) .
$$

This is replaced by

$$
V O L B(i 4)=V O L(i 4)+\left.\frac{\partial V^{t}}{\partial T}\right|_{P}\left(T^{B}-T^{t}\right) .
$$


- The $\operatorname{rgamma}(i 4)$ calculation has been removed. In its place, the calculation for $d v d p s(i 4)=\left.\frac{\partial V}{\partial P}\right|_{S}$ is performed, where,

$$
D V D P S=-\frac{V^{p}}{\rho^{p}} \frac{1}{C_{s}^{2}}
$$

as given in Eq. (32).

- The isentropic sound speed

$$
C_{s}^{2}=\left.\frac{\partial P}{\partial \rho}\right|_{S}=\left.\frac{\partial P}{\partial \rho}\right|_{T}+\frac{T}{C_{v} \rho^{2}}\left(\left.\frac{\partial P}{\partial T}\right|_{\rho}\right)^{2},
$$

used to calculate $d v d p s(i 4)$ above, is calculated based either on ideal gas or PR.

\section{WALLFILM.f and EVAP.f}

The modifications in these subroutines incorporate the following changes. Phase equilibrium is computed by equating liquid and vapor fugacities,

$$
f_{i}^{v}=f_{i}^{l} .
$$

For the Peng-Robinson EOS, fugacities are given by [7]

$$
\begin{gathered}
\ln \frac{f_{i}}{P x_{i}}=\frac{b_{i}}{b_{m}}(Z-1)-\ln \left(Z-B_{m}\right)- \\
\frac{A_{m}}{2 \sqrt{2} B_{m}}\left(\frac{2 \sum_{k=1}^{N} x_{k} a_{k i}}{a_{m}}-\frac{b_{i}}{b_{m}}\right) \ln \left(\frac{Z+2.414 B_{m}}{Z-0.414 B_{m}}\right),
\end{gathered}
$$

where

$$
A_{m}=\frac{a_{m} P}{R^{2} T^{2}}, \quad B_{m}=\frac{b_{m} P}{R T},
$$

and the compressibility factor $Z$ is calculated from [7]:

$$
Z^{3}-\left(1-B_{m}\right) Z^{2}+\left(A_{m}-3 B_{m}^{2}-2 B_{m}\right) Z-\left(A_{m} B_{m}-B_{m}^{2}-B_{m}^{3}\right)=0 .
$$

The smaller root of the cubic equation (36) applies to the liquid and the larger to the vapor.

We also correct for the latent heat of vaporization at high pressures using partial molar enthalpies $\bar{h}_{i}$. The molar latent heat of vaporization $W_{i} L_{i}$ is

$$
W_{i} L_{i}=\bar{h}_{i}^{v}-\bar{h}_{i}^{l}
$$

Partial molar enthalpies in each phase can be computed using [8]:

$$
\bar{h}_{i}-\bar{h}_{i}^{o}=-R T^{2} \frac{\partial}{\partial T} \ln \left(\frac{f_{i}}{P x_{i}}\right),
$$

where the superscript $o$ denotes the quantity in the ideal state. We correct for the pure fuel enthalpies using $[6]$ :

$$
W_{i}\left(h_{i}-h_{i}^{o}\right)=R T\left(Z_{i}-1\right)+\frac{\left(T \frac{d a_{i}}{d T}-a_{i}\right)}{(2 \sqrt{2}) b_{i}} \ln \left|\frac{Z_{i}+(1+\sqrt{2}) B_{i}}{Z_{i}+(1-\sqrt{2}) B_{i}}\right|,
$$

where $B_{i}=\frac{b_{i} p}{R T}$. 


\section{Additions to the Code}

\section{CVHEAT.f}

Calculates the specific heat based on Eq. (20). This is done for either an ideal or PR EOS.

\section{DELTAE.f}

Computes the internal energy departure based on Eq.(12), employing the PR EOS.

\section{DPDTRO.f}

Calculates the thermodynamic partial derivative $\left.\frac{\partial P}{\partial T}\right|_{\rho}$ using Eq. (13) corresponding to the PR EOS; i.e., this quantity is used to calculate the isentropic sound speed.

\section{DPDROT.f}

Calculates the thermodynamic partial derivative $\left.\frac{\partial P}{\partial \rho}\right|_{T}$ for the PR EOS, employing Eq. (14).

\section{EOSPROG.f}

Includes the computation of PR parameters $a_{m}, b_{m}$, and $\frac{d a_{m}}{d T}$. These are not only used in obtaining $\mathrm{P}-\mathrm{V}-\mathrm{T}$ relationships, but also in computing some partial derivatives.

\section{EOS.f}

Computes either the pressure, molar specific volume, or temperature of a gaseous mixture using the PR EOS.

\section{FRACMOLAR.f}

This subroutine is included to readily calculate molar fractions, and average molecular weight for mixtures. This information is needed before a call is made to EOS.f. We decided to include it as a separate subroutine to avoid writing duplicate code and to unclutter other subroutines. 



This report has been reproduced directly from the best available copy. It is available electronically on the Web (http://www.doe.gov/bridge).

Copies are available for sale to U.S. Department of Energy employees and contractors from:

Office of Scientific and Technical Information P.O. Box 62

Oak Ridge, TN 37831

(865) 576-8401

Copies are available for sale to the public from: National Technical Information Service

U.S. Department of Commerce

5285 Port Royal Road

Springfield, VA 22616

(800) 553-6847 
